\title{
Linear and Nonlinear Dynamics of North Atlantic Oscillations: A New Thinking of Symmetry Breaking
}

\author{
Dehai Luo And XiaOdan Chen \\ Key Laboratory of Regional Climate-Environment for Temperate East Asia, Institute of Atmospheric Physics, \\ Chinese Academy of Science, and University of Chinese Academy of Sciences, Beijing, China \\ STEVEN B. FELDSTEIN \\ Department of Meteorology and Atmospheric Science, The Pennsylvania State University, University Park, Pennsylvania
}

(Manuscript received 14 September 2017, in final form 25 February 2018)

\begin{abstract}
Observations reveal that the North Atlantic Oscillation (NAO) exhibits a strong asymmetry: large amplitude, long persistence, and westward movement in its negative phase $\left(\mathrm{NAO}^{-}\right)$and conversely in its positive phase $\left(\mathrm{NAO}^{+}\right)$. Further calculations show that blocking days occur frequently over the North Atlantic (Eurasia) after the $\mathrm{NAO}^{-}$ $\left(\mathrm{NAO}^{+}\right)$peaks, thus indicating that North Atlantic blocking occurs because of the retrogression of the $\mathrm{NAO}^{-}$, whereas blocking occurs over Eurasia because of enhanced downstream energy dispersion of the $\mathrm{NAO}^{+}$.

Motivated by a unified nonlinear multiscale interaction (UNMI) model, the authors define dispersion, nonlinearity, and movement indices to describe the basic characteristics of the NAO. On this basis, the physical cause of the strong asymmetry or symmetry breaking of the NAO is examined. It is revealed that the strong asymmetry between the $\mathrm{NAO}^{+}$and $\mathrm{NAO}^{-}$may be associated with the large difference of the North Atlantic jet in intensity and latitude between both phases. When the $\mathrm{NAO}^{+}$grows, the North Atlantic jet is intensified and shifts northward and corresponds to reduced nonlinearity and enhanced energy dispersion because of an increased difference between its group velocity and phase speed related to enhanced meridional potential vorticity gradient. Thus, the $\mathrm{NAO}^{+}$has smaller amplitude, eastward movement, and less persistence. Opposite behavior is seen for the $\mathrm{NAO}^{-}$because of the opposite variation of the North Atlantic jet during its life cycle. Thus, the above results suggest that the $\mathrm{NAO}^{+}\left(\mathrm{NAO}^{-}\right)$tends to be a linear (nonlinear) process as a natural consequence of the NAO evolution because of different changes in the North Atlantic jet between both phases.
\end{abstract}

\section{Introduction}

The North Atlantic Oscillation (NAO) is one of the most dominant and robust atmospheric low-frequency modes occurring in the North Atlantic. The NAO was first explicitly found by Exner (1913) and Walker (1923) from correlation maps and then widely studied by many modern meteorologists (Namias 1950; Lorenz 1951; Kutzbach 1970; Barnston and Livezey 1987; Ambaum and Hoskins 2002). The impact and mechanism of the $\mathrm{NAO}$ has been an important research topic, in part because changes in climate and extreme weather events in the Northern Hemisphere (NH) are often linked to the variability of the NAO (Hurrell 1995; Rogers 1997; Rodwell et al. 1999; Hurrell et al. 2003). In more recent years, a number of studies have focused on exploring the dynamics of the NAO, and significant progress has been

\footnotetext{
Corresponding author: Dr. Dehai Luo, ldh@mail.iap.ac.cn
}

made (Feldstein 2003; Vallis et al. 2004; Jin et al. 2006; Luo et al. 2007a,b, 2015; Rivière and Orlanski 2007; Woollings et al. 2008; Jiang et al. 2013; Dai et al. 2016; Song 2016). For example, it has been recognized that the NAO arises from Rossby wave breaking (Benedict et al. 2004; Franzke et al. 2004), is maintained by an eddy feedback (Hurrell 1995; Robinson 2000; Jin et al. 2006; Rivière and Orlanski 2007), and has a time scale of 10-20 days as seen from observational (Feldstein 2003) and theoretical (Luo et al. 2007a,b,c) aspects.

Using an idealized model, Luo et al. (2007a, 2015) showed that the occurrence of the NAO results from the evolution of preexisting synoptic-scale eddies and its phase is determined by the spatial structure of the preexisting planetary-scale dipole wave (Luo et al. 2007a, 2015). This

Publisher's Note: This article was revised on 14 June 2018 to correct a sign error in equation (3). 
viewpoint has been shown with numerical models by Song (2016). While the NAO shows an asymmetry in the occurrence frequency of North Atlantic blocking between its two phases (Scherrer et al. 2006; Croci-Maspoli et al. 2007), its two phases also exhibit a strong asymmetry at the decadal or interdecadal time scale in its zonal movement (Jung et al. 2003; Peterson et al. 2003; Cassou et al. 2004). In particular, Cassou et al. (2004) examined the asymmetry of the winter NAO pattern from a monthly mean perspective and attributed the interdecadal eastward shift of the positive NAO to increased greenhouse gas concentrations, supporting the finding of Ulbrich and Christoph (1999). However, in previous studies, less attention was focused on examining the physical cause of the zonal movement asymmetry for individual NAO life cycles with a 10-20-day time scale, although Watanabe (2004) has examined the asymmetric impact of the NAO on the Eurasian continent.

For individual NAO events with a 10-20-day time scale, Benedict et al. (2004) and Franzke et al. (2004) noted that there is an asymmetry of the wave breaking between both phases of the NAO during its life cycle. By extending the blocking model of Luo $(2000,2005)$, Luo et al. (2007a) proposed the unified nonlinear multiscale interaction (UNMI) model of NAO events, which showed that the daily evolution of individual NAO event exhibits strong asymmetries for the upstream and downstream circulation anomalies between the two phases of the NAO. Other studies also revealed that there is a strong asymmetry of the NAO persistence between the two phases of the NAO and attributed such persistence asymmetry to a large difference in the eddy vorticity forcing (Barnes and Hartmann 2010). Unfortunately, no previous studies have presented a unified viewpoint on the physical mechanism of the NAO asymmetry in persistence, zonal movement, and amplitude during the NAO life cycle. In this paper, we will first provide observational evidence to support that there is a strong asymmetry of the NAO amplitude and zonal movement during the NAO life cycle, as the anomalies associated with the negative NAO $\left(\mathrm{NAO}^{-}\right)$ are of large amplitude and move westward, and conversely for the positive NAO $\left(\mathrm{NAO}^{+}\right)$. For convenience, we will refer to the asymmetry of the NAO phases in terms of the flow pattern, persistence, zonal movement, and amplitude during the NAO life cycle as the symmetry breaking of NAO events. Such a definition is reasonable because the asymmetry of the NAO corresponds essentially to the breaking of its symmetry between both phases. On this basis, a new unified perspective is presented to account for the physical cause of the symmetry breaking of NAO events by defining dispersion, nonlinearity, and zonal movement indices, motivated by the UNMI model proposed by Luo et al. (2007a,b,c; 2015), and using reanalysis data. These indices defined from the daily evolution perspective can better represent the time variation of the NAO asymmetry than has been the case in previous studies, which can deepen our understanding of the physical cause of the symmetry breaking of the NAO.

This paper is organized as follows: In section 2, we describe the data, method, and model. In section 3, observational evidence is presented to support the perspective that the NAO has a strong energy dispersion asymmetry between its two phases through performing a composite analysis and defining an energy dispersion index. In section 4, we examine how the energy dispersion and nonlinearity vary with changes in the North Atlantic westerly wind to reveal the possible physical cause of the symmetry breaking of the NAO between its two phases. The conclusions and discussion are summarized in the final section.

\section{Data, method, and the model description}

\section{a. Data}

In this study, we use daily mean European Centre of Medium-Range Weather Forecasts (ECMWF) interim reanalysis (ERA-Interim) data for the winter season [from December to February (DJF)], for the period from December 1979-February 1980 to December 2015February 2016 (1979-2015) (Dee et al. 2011). The ERAInterim data are used to calculate NAO parameters such as the group velocity, zonal movement speed, dispersion, and nonlinearity. The primary variables that we use include daily 500-hPa geopotential height and zonal wind.

The daily NAO index in winter, based on the National Centers for Environmental Prediction-National Center for Atmospheric Research (NCEP-NCAR) reanalysis data, has been found to exhibit a correlation of 0.84 with that constructed from the daily $500-\mathrm{hPa}$ geopotential height field of the ERA-Interim data using the first empirical orthogonal function (EOF1) in the domain $90^{\circ} \mathrm{W}-40^{\circ} \mathrm{E}$ and $20^{\circ}-85^{\circ} \mathrm{N}$ (not shown). As noted below, $80 \%(78 \%)$ of the number of $\mathrm{NAO}^{+}\left(\mathrm{NAO}^{-}\right)$events based on the daily NAO index from the ERA-Interim data overlaps with that for the NOAA/CPC daily NAO index. This suggests that using ERA-Interim data to perform composites and to calculate the spatial variations of associated parameters is reasonable for the NAO even when the calculations are based on the daily CPCNAO index. It is preferable to use the NOAA/CPC NAO index, as that index has been used in numerous previous studies, and to use ERA-Interim data, which have a higher resolution than the NCEP-NCAR reanalysis data. Positive phase NAO $\left(\mathrm{NAO}^{+}\right)$and negative NAO 
$\left(\mathrm{NAO}^{-}\right)$phase events are defined as having taken place when the daily NAO index exceeds one standard deviation (1.0 STD) above and below its mean value for at least three consecutive days. The NAO events are discarded when the growth and decay of their NAO indices are nonmonotonic (Benedict et al.2004). We also define anomalies of 500-hPa geopotential height and zonal wind field as their deviation from the climatological mean field for each calendar day at each grid point.

\section{b. Blocking identification method}

An important feature of NAO variability is that the evolution of the $\mathrm{NAO}^{+}\left(\mathrm{NAO}^{-}\right)$event is often followed by changes in the frequency of downstream blocking events. To identify the spatial distribution and temporal variation of blocking events over Eurasia, we use the one-dimensional (1D) blocking index of Tibaldi and Molteni (1990), which is referred to as the 1D-TM index. The 1D-TM index is based on the meridional gradients of the 500-hPa geopotential height field at three fixed latitudes and has been extended in recent years to a twodimensional (2D) blocking index by many investigators such as Diao et al. (2006), Scherrer et al. (2006), and Davini et al. (2012). Here, we also used the 2D-TM index of Scherrer et al. (2006) and Davini et al. (2012) to examine the spatial distribution of blocking events from North Atlantic to Eurasia. The details of the 1D- and 2D-TM indices can be found in Tibaldi and Molteni (1990) and Davini et al. (2012), respectively. The 1D-TM index is convenient for the identification of the blocking events in a given domain, whereas the 2D-TM index of Scherrer et al. (2006) and Davini et al. (2012) is used to examine how the blocking is distributed over a wider region. For Ural (European) blocking, the calculation domain of the blocking ranges from $40^{\circ}$ to $80^{\circ} \mathrm{E}$ $\left(0^{\circ}\right.$ to $\left.40^{\circ} \mathrm{E}\right)$ when the $1 \mathrm{D}-\mathrm{TM}$ index is used to identify the blocking events. For the spatial distribution of the blocking frequency from the North Atlantic to Eurasia, we confine the calculation region to the latitude and longitude ranges of $30^{\circ}-75^{\circ} \mathrm{N}$ and $90^{\circ} \mathrm{W}-90^{\circ} \mathrm{E}$ when the $2 \mathrm{D}-\mathrm{TM}$ index is used.

\section{c. UNMI model description}

Although previous models can capture the time-mean NAO pattern (Vallis et al. 2004; Jin et al. 2006), they are unable to represent the life cycle of an NAO event with a time scale of 10-20 days (Feldstein 2003). Thus, these models cannot be used to investigate the asymmetry of the NAO between its two phases, especially its persistence and zonal movement. This motivates us to use the UNMI model (Luo et al. 2007a,b,c, 2015) to describe the life cycle of an NAO event. The UNMI model is an extension of the nonlinear multiscale interaction (NMI) model of blocking events, proposed in Luo (2000, 2005), Luo and Li (2000), and Luo et al. (2014). This UNMI model provides a useful tool for examining why the NAO exhibits a strong asymmetry between its two phases during its evolution.

The UNMI model is based on the zonal-scale separation assumption between the NAO anomaly with its low zonal wavenumber and synoptic-scale eddies with higher zonal wavenumbers (Luo 2000, 2005; Luo and Li 2000). Because the NAO pattern is approximately equivalent barotropic in the troposphere (Feldstein 2003; Vallis et al. 2004; Jin et al. 2006), it is reasonable to use the equivalent barotropic potential vorticity (PV) model as used in Luo and Li (2000), Luo (2005), and Luo et al. $(2007 \mathrm{a}, 2015)$ to examine the problems mentioned in introduction.

We decompose the nondimensional barotropic atmospheric streamfunction $\psi_{T}$ into three parts: prior basic flow $\bar{\psi}(y)=-u_{0} y\left(u_{0}\right.$ is the basic westerly wind prior to the NAO onset and assumed to be a constant), large-scale streamfunction anomaly $\psi$ with zonal wavenumber $k$, and synoptic-scale eddies $\psi^{\prime}$ with zonal wavenumber $\tilde{k}_{j}(j=1,2,3, \ldots)$ in the form of $\psi_{T}=\bar{\psi}(y)+$ $\psi+\psi^{\prime}$. Here, $\psi$ is assumed to include two parts: mean westerly wind change and NAO anomaly. When the scale separation assumption $k \ll \tilde{k}_{j}$ (Luo 2000,2005) is made, the planetary- and synoptic-scale PV equations of the UNMI model derived from a nondimensional barotropic PV equation are obtained as (Luo and Li 2000; Luo et al. 2001, 2014; Luo 2005; Luo et al. 2007a,b,c, 2015)

$$
\begin{aligned}
& \left(\frac{\partial}{\partial t}+u_{0} \frac{\partial}{\partial x}\right)\left(\nabla^{2} \psi-F \psi\right)+J\left(\psi, \nabla^{2} \psi\right)+\left(\beta+F u_{0}\right) \frac{\partial \psi}{\partial x} \\
& \quad=-\nabla \cdot\left(\mathbf{v}^{\prime} q^{\prime}\right)_{P}, \\
& \left(\frac{\partial}{\partial t}+u_{0} \frac{\partial}{\partial x}\right)\left(\nabla^{2} \psi^{\prime}-F \psi^{\prime}\right)+\left(\beta+F u_{0}\right) \frac{\partial \psi^{\prime}}{\partial x} \\
& \quad=-J\left(\psi^{\prime}, \nabla^{2} \psi\right)-J\left(\psi, \nabla^{2} \psi^{\prime}\right)+\nabla^{2} \psi_{S}^{*},
\end{aligned}
$$

where the characteristic scale $L=10^{3} \mathrm{~km}$ and wind speed $U_{0}=10 \mathrm{~m} \mathrm{~s}^{-1}$ have been used in the derivation of Eq. (1), $F=\left(L / R_{d}\right)^{2}, \beta=\beta_{0} L^{2} / U_{0}, \beta_{0}$ is the meridional gradient of the Coriolis parameter at a given latitude $\phi_{0}$, and $R_{d} \approx L$ is the radius of Rossby deformation. Note that terms involving $F$ denote the divergence term associated with barotropic motions when $R_{d}$ is finite and is close to zero and represents a nondivergent flow when $R_{d} \rightarrow \infty$. The quantity $\nabla \cdot\left(\mathbf{v}^{\prime} q^{\prime}\right)_{P}=J\left(\psi^{\prime}, \nabla^{2} \psi^{\prime}\right)_{P}$ denotes the planetary-scale component of $\nabla \cdot\left(\mathbf{v}^{\prime} q^{\prime}\right)$ induced by synoptic eddies, which has a zonal wavenumber close to that of the NAO anomaly, $\mathbf{v}^{\prime}=\left(u^{\prime}, v^{\prime}\right)=$ $\left(-\partial \psi^{\prime} / \partial y, \partial \psi^{\prime} / \partial x\right)$ is the horizontal wind vector of the 
synoptic-scale eddies with its relative vorticity $q^{\prime}=\nabla^{2} \psi^{\prime}$, and $\nabla^{2} \psi_{S}^{*}$ is the synoptic-scale vorticity source term (i.e., a high-frequency wavemaker) and is designed to maintain preexisting synoptic-scale eddies prior to the NAO onset. The mathematical expression of $\psi_{S}^{*}=\psi_{S}^{*}(x$, $y, t)$ can be found in Luo (2005). Remaining notations can be found in Luo et al. $(2014,2015)$. The merit of this UNMI model is that it is able to describe under what conditions $\mathrm{NAO}^{+}$or $\mathrm{NAO}^{-}$events can occur and why these events show 10-20-day variability (Luo et al. 2007a). This model shows that the occurrence of an $\mathrm{NAO}^{+}$or $\mathrm{NAO}^{-}$event depends crucially on if the PV field of the initial NAO matches the spatial structure of $-\nabla \cdot\left(\mathbf{v}^{\prime} q^{\prime}\right)_{P}$, induced by preexisting synoptic-scale eddies. When the preexisting planetary-scale dipole anomaly has a positive-over-negative pattern and the preexisting structure of $-\nabla \cdot\left(\mathbf{v}^{\prime} q^{\prime}\right)_{P}$ shows a negative-over-positive dipole pattern, Eq. (1) can describe the life cycle of a blocking dipole or $\mathrm{NAO}^{-}$ pattern. This model is the so-called NMI model (Luo 2000, 2005; Luo and Li 2000; Luo et al. 2014). When we include the $\mathrm{NAO}^{+}$component in the NMI model, this model becomes an extended form of the NMI model and is referred to as the UNMI model (Luo et al. 2007a, 2015).

In the models of Shutts (1983) and Haines and Marshall (1987), the general method used to derive the mean flow-eddy interaction is the time decomposition with two different time scales. If one uses the time decomposition in the form of $\psi_{T}=-u_{0} y+\bar{\psi}+\psi^{\prime}$, where $u_{0}$ is the same as above, $\bar{\psi}$ is a time-mean streamfunction anomaly averaged over a duration longer than $\tau$, and $\psi^{\prime}$ is the streamfunction anomaly of the synoptic transient eddy with a short period of $\tau$, then the PV equations of the NAO anomaly-transient eddy interaction can be obtained as

$$
\begin{aligned}
& \left(\frac{\partial}{\partial t}+u_{0} \frac{\partial}{\partial x}\right)\left(\nabla^{2} \bar{\psi}-F \bar{\psi}\right)+J\left(\bar{\psi}, \nabla^{2} \bar{\psi}\right)+\left(\beta+F u_{0}\right) \frac{\partial \bar{\psi}}{\partial x}=-\nabla \cdot\left(\overline{\mathbf{v}^{\prime} q^{\prime}}\right) \\
& \left(\frac{\partial}{\partial t}+u_{0} \frac{\partial}{\partial x}\right)\left(\nabla^{2} \psi^{\prime}-F \psi^{\prime}\right)+\left(\beta+F u_{0}\right) \frac{\partial \psi^{\prime}}{\partial x}+J\left(\psi^{\prime}, \nabla^{2} \psi^{\prime}\right) \\
& \quad=\nabla \cdot\left(\overline{\mathbf{v}^{\prime} q^{\prime}}\right)-J\left(\psi^{\prime}, \nabla^{2} \bar{\psi}\right)-J\left(\bar{\psi}, \nabla^{2} \psi^{\prime}\right)+\nabla^{2} \psi_{S}^{*},
\end{aligned}
$$

where the overbar denotes a time average.

It is difficult to obtain an analytical solution of Eq. (2) unless $\nabla \cdot\left(\overline{\mathbf{v}^{\prime} q^{\prime}}\right)$ is given, because it is a time-mean quantity. Also, this equation cannot tell us what is the difference between the persistence and zonal movement for the two phases of the NAO because $\bar{\psi}$ shows only a time-mean structure. In this case, it is difficult to use Eq. (2) to examine the asymmetry of the circulation, zonal movement, and persistence for the NAO. It is also seen that Eq. (2) reduces to the form used by Haines and Marshall (1987) when $J\left(\psi^{\prime}, \nabla^{2} \psi^{\prime}\right)=\nabla \cdot\left(\overline{\mathbf{v}^{\prime} q^{\prime}}\right)$ is assumed. To obtain the solution of Eq. $(2 \mathrm{a}), \nabla \cdot\left(\overline{\mathbf{v}^{\prime} q^{\prime}}\right)$ can be parameterized as a known function as in Vallis et al. (2004) and also as a function proportional to $\bar{\psi}$ as in Jin et al. (2006). For these cases, the solution is time independent and cannot be used to explore the daily evolution of the NAO. However, using Eq. (1) in a UNMI model can avoid this difficulty because $\nabla \cdot\left(\mathbf{v}^{\prime} q^{\prime}\right)_{P}$ is time dependent. In the previous studies of Luo $(2000,2005)$ and Luo et al. (2001, 2007a), the NMI model, or its extended form (the UNMI model), obtained from Eq. (1), has been used to examine the daily evolution of blocking and NAO events by deriving an analytical solution under the assumptions that the synoptic transient eddy and blocking or NAO anomaly are zonally separated and the synoptic-scale eddies are composed of preexisting incident synoptic-scale eddies $\psi_{1}^{\prime}$ and deformed synoptic-scale eddies $\psi_{2}^{\prime}$ (Luo 2000, 2005; Luo and Li 2000; Luo et al. 2014). In this UNMI model, $\psi_{1}^{\prime}$ is prespecified in the form of a known function independent of the NAO evolution, whereas $\psi_{2}^{\prime}$ is not prespecified and corresponds to deformed eddies induced by the NAO feedback, which depends on the temporal evolution of the subsequent NAO pattern. The analytical solution of the UNMI model can be obtained by using the multiple-scale method (Luo et al. 2007a,c, 2015), and the model solution can be used to describe how the NAO pattern changes with time if its initial condition is prespecified. This model provides an efficient tool for examining why the NAO shows strong asymmetry between its two phases. Below, we only briefly describe the analytical solution of the UNMI model and use it to construct the dispersion, nonlinearity, and zonal movement indices to represent the basic features of the observed NAO.

Here, we consider a quasi-stationary planetary-scale anomaly having a meridional dipole structure with both meridional wavenumber $m$ and zonal wavenumber $k$ as the NAO anomaly, and the preexisting synoptic-scale 
eddies $\psi_{1}^{\prime}$ are assumed to have a meridional monopole structure and consist of two zonal wavenumbers $\tilde{k}_{1}$ and $\tilde{k}_{2}$ corresponding to two different frequencies of $\tilde{\omega}_{1}$ and $\tilde{\omega}_{2}$, respectively. In this case, the analytical solution of the total atmospheric streamfunction $\left(\psi_{T}\right)$ of an NAO event derived from Eq. (1) can be expressed as in Luo et al. (2007a, 2015); that is,

$$
\begin{aligned}
& \psi_{T}=-u_{0} y+\psi+\psi^{\prime}=\psi_{P}+\psi^{\prime}, \\
& \psi_{P}=-u_{0} y+\psi \approx-u_{0} y+\psi_{\mathrm{NAO}}+\psi_{m},
\end{aligned}
$$

$$
\begin{aligned}
\psi_{\mathrm{NAO}}= & B \sqrt{\frac{2}{L_{y}}} \exp [i(k x-\omega t)] \sin (m y)+\mathrm{cc}, \\
\psi_{m}= & -|B|^{2} \sum_{n=1}^{\infty} q_{n} g_{n} \cos \left(n+\frac{1}{2}\right) m y, \\
\psi^{\prime} \approx & \psi_{1}^{\prime}+\psi_{2}^{\prime}, \\
\psi_{1}^{\prime}= & f_{0}(x)\left\{\exp \left[i\left(\tilde{k}_{1} x-\tilde{\omega}_{1} t\right)\right]\right. \\
& \left.+\alpha \exp \left[i\left(\tilde{k}_{2} x-\tilde{\omega}_{2} t\right)\right]\right\} \sin \left(\frac{m}{2} y\right)+\mathrm{cc},
\end{aligned}
$$

where $\Delta \omega=\tilde{\omega}_{2}-\tilde{\omega}_{1}-\omega, \omega=u_{0} k-\left[\left(\beta+F u_{0}\right) k\right] /\left(k^{2}+\right.$ $\left.\left.m^{2}+F\right)\right], \tilde{\omega}_{j}=u_{0} \tilde{k}_{j}-\left[\left(\beta+F u_{0}\right) \tilde{k}_{j}\right] /\left(\tilde{k}_{j}^{2}+m^{2} / 4+F\right), j=1$ and $2, \Delta k=k-\left(\tilde{k}_{2}-\tilde{k}_{1}\right),|B|^{2}=B B^{*}, C_{g}=u_{0}-\left(\beta+F u_{0}\right)$ $\left(m^{2}+F-k^{2}\right) /\left(k^{2}+m^{2}+F\right)^{2}, k=2 k_{0}, k_{0}=1 /\left(6.371 \cos \phi_{0}\right)$, $\lambda=\left[3\left(m^{2}+F\right)-k^{2}\right]\left(\beta+F u_{0}\right) /\left(m^{2}+F+k^{2}\right)^{3}$, $\delta=\delta_{N} /\left(\beta+F u_{0}\right), \delta_{N}=k m \sum_{n=1}^{\infty} q_{N_{n}} g_{n}^{2}\left[k^{2}+m^{2}-\right.$ $\left.m^{2}(n+1 / 2)^{2}\right] /\left(m^{2}+F+k^{2}\right), G=\alpha\left(L_{y} / 2\right)^{1 / 2}\left(\tilde{k}_{1}+\tilde{k}_{2}\right)^{2}$ $\left(\tilde{k}_{2}-\tilde{k}_{1}\right) /\left[4\left(m^{2}+F+k^{2}\right)\right], m= \pm 2 \pi / L_{y}, g_{n}=$ $8 /\left\{m[4-(n+1 / 2)] L_{y}\right\}, q_{N_{n}}=4 k^{2} m /\left(L_{y}\left\{1-\left(m^{2}+F-k^{2}\right)\right.\right.$ $\left.\left.\left[F+m^{2}(n+1 / 2)^{2}\right] /\left(m^{2}+F+k^{2}\right)^{2}\right\}\right), \delta>0, \delta_{N}>0$, and cc denotes the complex conjugate of its preceding term.

In Eq. (3), $\omega$ is the frequency of the NAO pattern with meridional wavenumber $m, \phi_{0}$ is the reference latitude, $L_{y}$ is the width of the nondimensional beta plane channel, $\alpha_{1}=1$ and $\alpha_{2}=\alpha= \pm 1$, and $f_{0}(x)$ is the spatial distribution of the amplitude of the preexisting synopticscale eddies with two frequencies $\tilde{\omega}_{j}(j=1,2)$. The remaining details of the other coefficients and the notation can be found in Luo and Li (2000), Luo (2005), and Luo et al. (2007a,b, 2014). Moreover, $\psi_{\text {NAO }}$ is the NAO streamfunction anomaly, and $\psi_{m}$ is the streamfunction of the mean zonal wind change due to the presence of the NAO anomaly, while the zonal wind prior to the NAO onset is assumed to be a constant. Note that $B$ represents the complex amplitude of the NAO anomaly with its complex conjugate $B^{*}$, which varies in time and in the zonal direction and is described by a forced nonlinear Schrödinger (NLS) Eq. (3h). It is worth pointing out that in Eq. (3h), $C_{g}$ is the group velocity, $\lambda$ denotes the linear dispersion term, and $\delta$ is positive for $u_{0}>-\beta / F$ and is the nonlinearity coefficient as a measure of the nonlinearity strength.

When $\alpha<0$ and $m<0$, the solution (3) describes the life cycle of a blocking event (Luo 2000, 2005; Luo et al. 2014; Luo and Li 2000) or an $\mathrm{NAO}^{-}$event (Luo et al. 2007a,b,c). Thus, the solution (3) with $\alpha<0$ and $m<$ 0 represents a blocking or $\mathrm{NAO}^{-}$flow and its interaction with synoptic-scale eddies and is referred to as an NMI model as noted above. But when $\alpha>0$ and $m>0$, the solution (3) represents the life cycle of an $\mathrm{NAO}^{+}$event (Luo et al. 2007a,b,c). In this case, Eq. (1) actually represents a UNMI model when $\alpha$ and $m$ are chosen as positive and negative values in the solution (3).

One merit of this UNMI model is its ability to unify blocking and NAO events into a single model. Another advantage of the UNMI model is its ability to describe the relationship between the phase of the NAO and downstream blocking. For this UNMI model, we can rewrite Eq. (1a) as $\partial q / \partial t+J\left(-u_{0}+\psi, \nabla^{2} \psi+\beta y\right)=-\nabla \cdot\left(\mathbf{v}^{\prime} q^{\prime}\right)_{P}$, where $\mathbf{v}^{\prime}=\mathbf{v}_{1}^{\prime}+\mathbf{v}_{2}^{\prime}, \quad q^{\prime}=q_{1}^{\prime}+q_{2}^{\prime}, \quad q_{j}^{\prime}=\nabla^{2} \psi_{j}^{\prime}$, and $\mathbf{v}_{j}^{\prime}=\mathbf{i}\left(-\partial \psi_{j}^{\prime} / \partial y\right)+\mathbf{j}\left(\partial \psi_{j}^{\prime} / \partial x\right)(j=1,2)$. Because $J\left(-u_{0}+\psi\right.$, $\left.\nabla^{2} \psi+\beta y\right) \approx 0$ holds for blocking (Shutts 1983) and the NAO (Luo et al. 2007b), one obtains $\partial q / \partial t \approx-\nabla \cdot\left(\mathbf{v}^{\prime} q^{\prime}\right)_{P}$. During the initial stage $(t \sim 0)$ of the NAO, we have $\mathrm{v}_{2}^{\prime} \approx 0$ and $q_{2}^{\prime} \approx 0$ because its amplitude is extremely small. In this case, it is found that $\partial q / \partial t \approx-\nabla \cdot\left(\mathbf{v}_{1}^{\prime} q_{1}^{\prime}\right)_{P}$. Thus, it is clear that the initial growth of the NAO is mainly determined by the preexisting incident synoptic-scale eddies $\psi_{1}^{\prime}$ 
rather than by the deformed eddies $\psi_{2}^{\prime}$. When $-\nabla \cdot\left(\mathbf{v}_{1}^{\prime} q_{1}^{\prime}\right)_{P}$ induced by the preexisting incident synoptic-scale eddies $\psi_{1}^{\prime}$ matches the PV field of the incipient NAO anomaly with a small nonzero initial amplitude, the preexisting incident synoptic-scale eddies $\psi_{1}^{\prime}$ can amplify the incipient NAO to excite an NAO with a realistic amplitude. This is the so-called eddy-NAO matching mechanism proposed by Luo et al. (2007a, 2015). This mechanism is different from the eddy-straining mechanism of Shutts (1983) and wave-breaking viewpoint of Benedict et al. (2004), Franzke et al. (2004), and Woollings et al. (2008). This result has been verified by using the conditional nonlinear optimal perturbation (CNOP) method of Mu and Jiang (2008) and the recent numerical experiment of Song (2016). On the other hand, we mention that the synoptic-scale eddy variation tends to have the same low-frequency time scale as that of the NAO because the deformed eddies include the amplitude of the NAO. This means that the time scales of the synoptic-scale eddies and NAO anomaly cannot be separated, though their spatial scales are separated. This also suggests that the time decomposition used to derive Eq. (2) is likely problematic once the synoptic-scale eddies and NAO anomaly interact with each other. This is a main reason why we use the UNMI model to examine the physical mechanism that drives the symmetry breaking of the NAO in this study.

In this paper, we will use the reanalysis data to examine what causes the asymmetry of the NAO between its two phases by defining indices motivated by the NAO amplitude Eq. (3h). This will be done in the following sections.

\section{Analysis results on the symmetry breaking of the NAO}

Before examining the features of the symmetry breaking of the NAO, it is useful to determine the number of NAO events during 1979-2015. This calculation shows that for a one standard deviation definition of the NOAA/CPC NAO index, which is based on NCEP-NCAR reanalysis data, there are $55 \mathrm{NAO}^{+}$and $27 \mathrm{NAO}^{-}$events in winter during 1979-2015 and $94 \mathrm{NAO}^{+}$and $67 \mathrm{NAO}^{-}$events during 1950-2015. It is also found that there are $52 \mathrm{NAO}^{+}$ and $33 \mathrm{NAO}^{-}$events during 1979-2015 based on the ERA-Interim data if the EOF1 of the daily $500-\mathrm{hPa}$ geopotential height anomaly in the domain $90^{\circ} \mathrm{W}-40^{\circ} \mathrm{E}$ and $20^{\circ}-85^{\circ} \mathrm{N}$ is used to define the daily NAO index. The calculation shows that $80 \%(78 \%)$ of the number of $\mathrm{NAO}^{+}\left(\mathrm{NAO}^{-}\right)$events overlaps for the two reanalysis datasets. In this paper, we use the NAO events selected based on the NCEP-NCAR data during 1979-2015 to examine the basic features of the NAO pattern between its two phases, though the composite of the geopotential height anomaly is based on the ERA-Interim data. We also show the composite daily 500-hPa geopotential height anomalies in Fig. 1 for the two phases of the NAO event. It is found that the $\mathrm{NAO}^{-}$(Fig. 1b) has larger maximum amplitude than the $\mathrm{NAO}^{+}$(Fig. 1a). Here, we only consider the zonal movement of the northern pole of the NAO because the amplitude of the northern pole is much larger than that of the southern pole. Such an approach has been used in Wang and Magnusdottir (2012). Moreover, the $\mathrm{NAO}^{-}\left(\mathrm{NAO}^{+}\right)$is also seen to undergo clear westward (eastward) movement as shown in Fig. 2 for its timelongitude evolution when performing a meridional average of the daily 500-hPa geopotential height anomaly between $50^{\circ}$ and $80^{\circ} \mathrm{N}$. This feature has been noted by Luo et al. (2012), which is different from the result of Baxter and Nigam (2013), who found a westward shift of the $\mathrm{NAO}^{+}$based on the EOF analysis by assuming the two NAO phases were symmetric. In particular, the westward movement of the $\mathrm{NAO}^{-}$is slightly faster than the eastward movement of the $\mathrm{NAO}^{+}$(Fig. 2). This reflects the asymmetry of the NAO movement between its two phases. It needs to be pointed out that the zonal movement of the southern pole of the NAO is less evident because its amplitude and associated zonal wind change are smaller (not shown). In summary, the above results reflect a strong asymmetry of the NAO pattern in amplitude and longitudinal movement between its two phases.

As further seen in Fig. 1, the occurrence of the $\mathrm{NAO}^{+}$ $\left(\mathrm{NAO}^{-}\right)$dipole originates from the growing of an eastward (westward)-moving cyclonic (anticyclonic) circulation centered over Greenland (Northern Europe) (see Fig. 1 for lag -8 days). An interesting result we find here is that for the $\mathrm{NAO}^{+}$, the wave train structure from the North Atlantic to the Barents-Kara Seas tends to be more evident after its peak (Fig. 1a from lag 0 to 4 days) rather than before. Especially after lag 4 days, the $\mathrm{NAO}^{+}$ rapidly decays. In contrast, a wave train structure for the $\mathrm{NAO}^{-}$is hardly seen during its life cycle. This suggests that the $\mathrm{NAO}^{+}$more easily disperses its energy in a downstream direction than does the $\mathrm{NAO}^{-}$. Figures $3 \mathrm{a}, \mathrm{b}$ show the time series of the composite daily $\mathrm{NAO}$ index for $\mathrm{NAO}^{+}$and $\mathrm{NAO}^{-}$events during 1979-2015 (Fig. 3a) and 1950-2015 (Fig. 3b). Here, the duration of the NAO is defined based on the $e$-folding time scale of the daily NAO index, which is the days when the amplitude of the NAO index exceeds the NAO index value divided by $e$. It can be seen for 1979-2015 that $\mathrm{NAO}^{-}$events are more persistent than $\mathrm{NAO}^{+}$events (Fig. 3a) as the mean duration of the $\mathrm{NAO}^{+}$and $\mathrm{NAO}^{-}$ events are 10 and 13 days, respectively. Furthermore, the difference between the mean duration (i.e., 3 days) is statistically significant at the $95 \%$ confidence level for a Monte Carlo test with 5000 simulations. During 

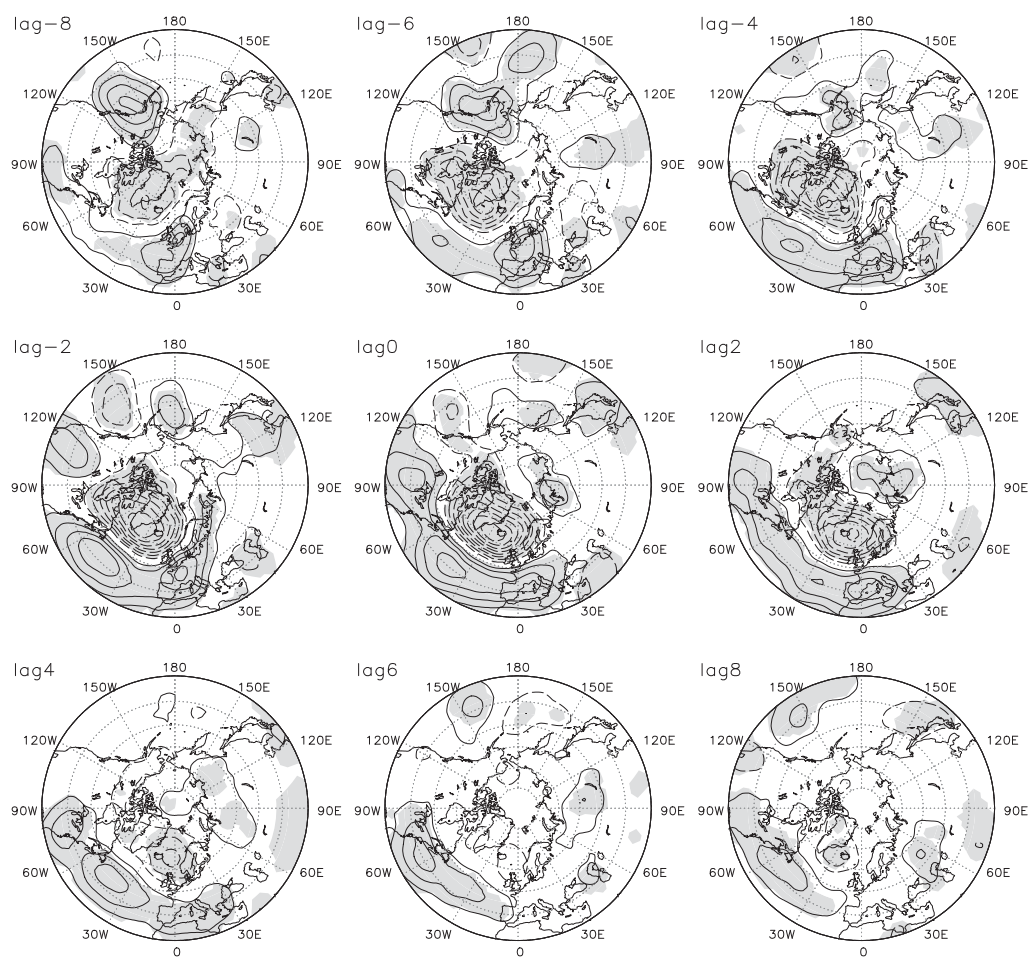

(a)
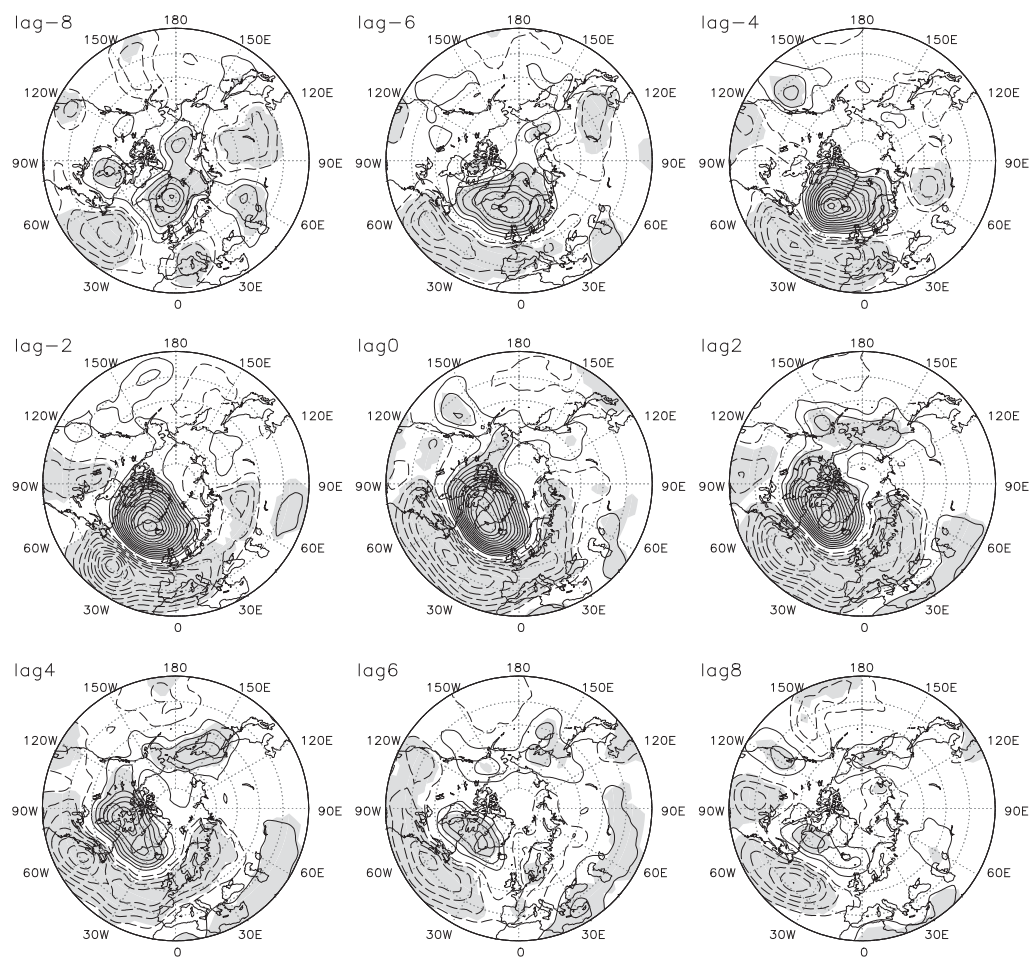

(b)

FIG. 1. Instantaneous fields of composite daily 500-hPa geopotential height anomalies $\left(\mathrm{CI}=20 \mathrm{gpm}\right.$ ) for (a) $\mathrm{NAO}^{+}$and (b) $\mathrm{NAO}^{-}$events during 1979-2015 from lags -8 to +8 days; lag 0 denotes the peak day of the NAO anomaly for its two phases. The gray shading denotes the region above the $95 \%$ confidence level for the two-sided Student's $t$ test. 
(a)

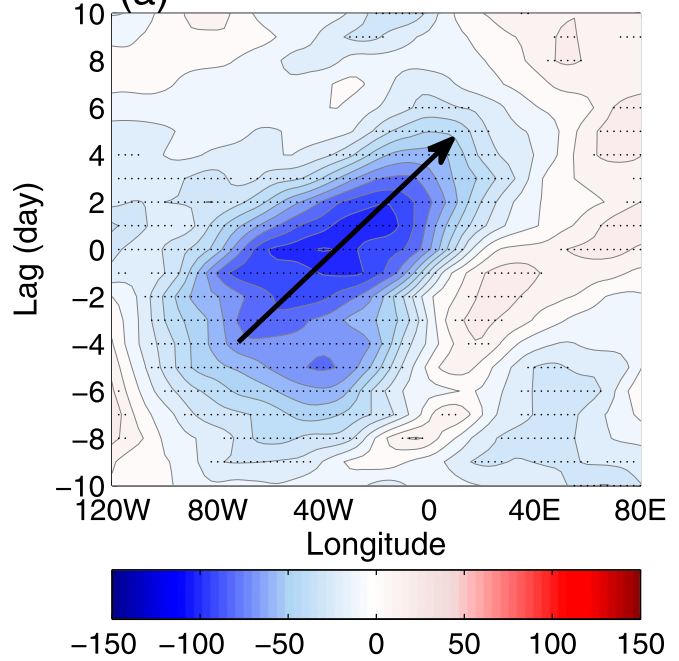

(b)

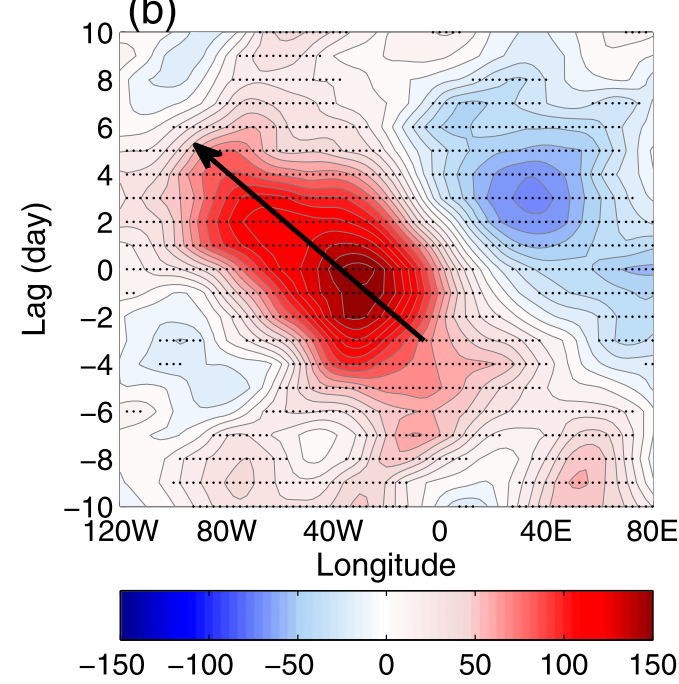

FIG. 2. Time-longitude evolution of latitude-averaged composite daily 500-hPa geopotential height anomalies (gpm) over $50^{\circ}-80^{\circ} \mathrm{N}$ during the NAO life cycle for (a) $\mathrm{NAO}^{+}$and (b) $\mathrm{NAO}^{-}$events during 1979-2015. Lag 0 denotes the peak day of the NAO. The dotted regions are significant above the $95 \%$ confidence level for the two-sided Student's $t$ test, and the arrow represents the movement direction of the NAO anomaly.

1950-2015, $\mathrm{NAO}^{+}\left(\mathrm{NAO}^{-}\right)$events have a mean duration of 9 (11) days, and the mean duration difference between the $\mathrm{NAO}^{+}$and $\mathrm{NAO}^{-}$is statistically significant at the 90\% confidence level (Fig. 3b), again based on a Monte Carlo test. Moreover, the $\mathrm{NAO}^{-}$has larger amplitude (blue line in Figs. 3a,b) than the $\mathrm{NAO}^{+}$(red lines in Figs. 3a,b). The amplitude difference between the two phases of the NAO is statistically significant at the $95 \%$ confidence level for a Monte Carlo test. This indicates that the NAO shows a strong asymmetry between its two phases in duration and amplitude. Moreover, we also tested the sensitivity of the results to the selection criteria of the NAO events. For example, during 1950-2015, there are $45 \mathrm{NAO}^{+}$and $41 \mathrm{NAO}^{-}$events, when an $\mathrm{NAO}^{+}$ $\left(\mathrm{NAO}^{-}\right)$event is defined to take place if the daily $\mathrm{NAO}$ index exceeds the threshold of the upper 95th (lower 5th) quantile (Palmer and Räisänen 2002; Yiou and Nogaj 2004) for at least three consecutive days. When using NAO events based on the percentile approach, the difference between the durations of the $\mathrm{NAO}^{+}$and $\mathrm{NAO}^{-}$ (10 and 12 days, respectively) is statistically significant at the $90 \%$ confidence level for a Monte Carlo test (not shown). Below, we will indicate that the NAO asymmetry is likely attributed to a large difference in the dispersion and nonlinearity between the $\mathrm{NAO}^{+}$and $\mathrm{NAO}^{-}$because of North Atlantic westerly wind changes.

To further see the change in dispersion during the evolution of the NAO, we show the time-mean composite of the daily 500-hPa geopotential height anomalies averaged from lag -5 to 0 days (growth) and from lag 0 to 5 days (decay) in Fig. 4 for $\mathrm{NAO}^{+}$and $\mathrm{NAO}^{-}$ events during 1950-2015 (lag 0 denotes the peak of the NAO). For both phases of the NAO, it is seen that the wave train structure is more pronounced during the decay phase (Figs. 4b and 4d) than during the growth phase (Figs. $4 \mathrm{a}$ and $4 \mathrm{c}$ ). In fact, whether the NAO shows strong dispersion is mainly determined by the relative strength between the northern anomaly of NAO dipole and the closest anomaly downstream of the NAO region. To quantify this dispersion change, here we define $D_{I}=\left(H_{A}-H_{D}\right) / H_{A}$ as a daily energy dispersion index for the NAO, where $H_{A}$ denotes the absolute value of the domain-mean daily $500-\mathrm{hPa}$ geopotential height anomaly averaged over an area of $4^{\circ}$ latitude $\times 4^{\circ}$ longitude around the anticyclonic (cyclonic) center of the $\mathrm{NAO}^{-}\left(\mathrm{NAO}^{+}\right)$in the North Atlantic (i.e., the northern anomaly of the NAO dipole). But in the expression for $D_{I}, H_{D}$ is defined as the domain-mean daily geopotential height anomaly averaged over an area of $4^{\circ}$ latitude $\times$ $4^{\circ}$ longitude around the cyclonic (anticyclonic) center in the downstream side (Eurasia) of the North Atlantic for the $\mathrm{NAO}^{-}\left(\mathrm{NAO}^{+}\right)$. How the $D_{I}$ index is constructed is described in the appendix for the $\mathrm{NAO}^{-}$. This index reflects the change in magnitude of the geopotential height anomaly from the upstream NAO region to farther downstream and the energy dispersion of the NAO. The NAO is understood to be weakly dispersive if $D_{I}$ is strongly positive. In contrast, the NAO is strongly dispersive if $D_{I}$ is weakly positive or strongly negative. In this case, it is straightforward to use the value of $D_{I}$ to judge whether the NAO exhibits strong dispersion. While $D_{I}$ is almost the same for both the $\mathrm{NAO}^{+}$and $\mathrm{NAO}^{-}$before 

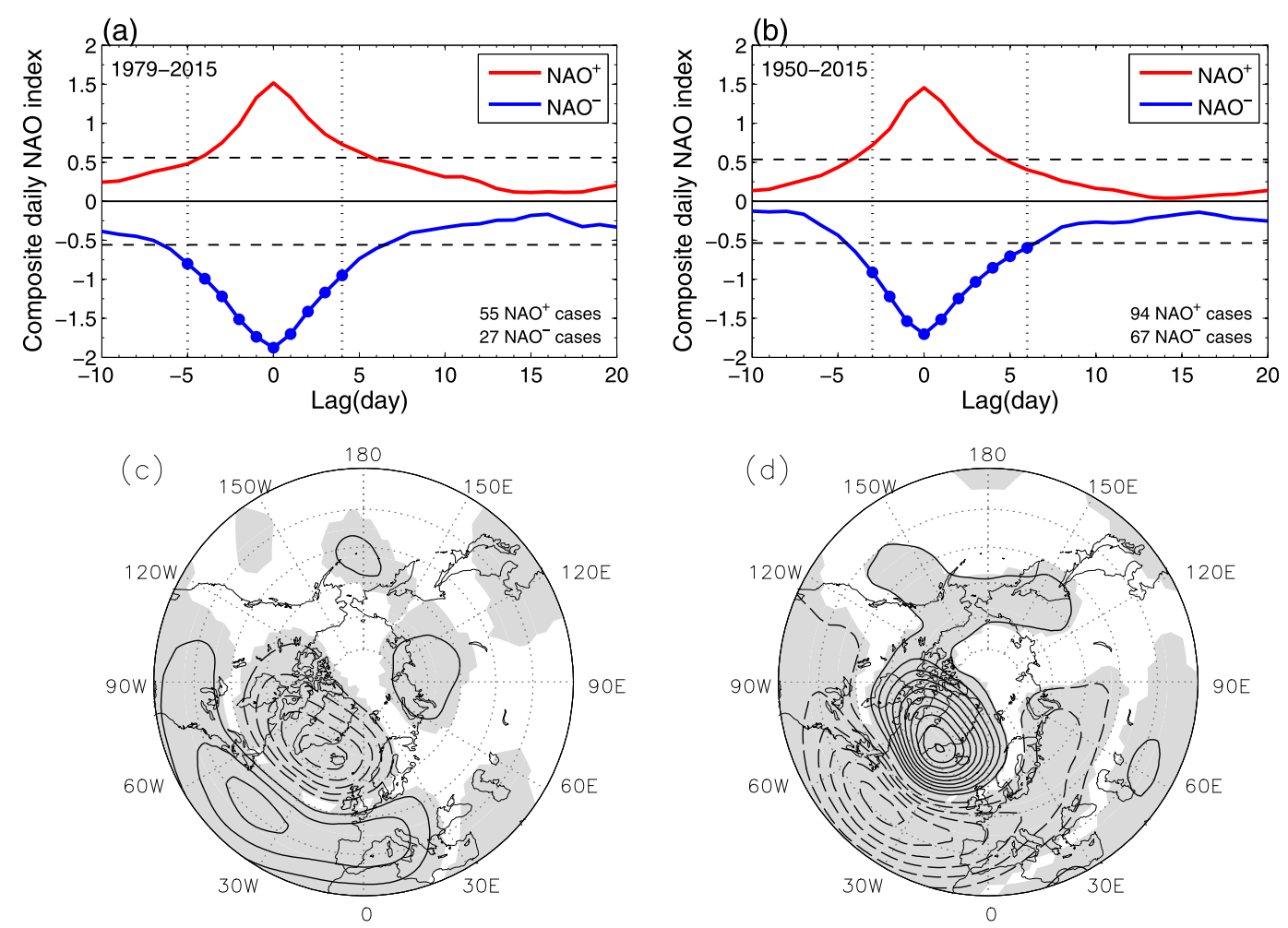

FIG. 3. (a),(b) Composites of daily NAO indices for $\mathrm{NAO}^{+}$(red solid line) and $\mathrm{NAO}^{-}$(blue solid line) events during (a) 1979-2015 and (b) 1950-2015, where the dashed line represents the $e$-folding line of the NAO ${ }^{+}$index and the blue dots denote the lag when the difference of the NAO amplitude between the two phases of the NAO is statistically significant at the $95 \%$ confidence level for a Monte Carlo test with a 5000-times simulation. (c),(d) The time-mean daily composite $500-\mathrm{hPa}$ geopotential height anomalies $(\mathrm{CI}=20 \mathrm{gpm})$ averaged from lag -5 to 5 days for $(\mathrm{c}) \mathrm{NAO}^{+}$and (d) $\mathrm{NAO}^{-}$events during 1979-2015. Lag 0 denotes the day of the NAO peak for its two phases. The black dashed lines in (a),(b) represent the $e$-folding value of the $\mathrm{NAO}^{+}$index, and the black solid line denotes the zero line. In (c) and (d), the gray shading denotes the region above the $95 \%$ confidence level for the two-sided Student's $t$ test.

lag +2 days (Fig. 4e), it becomes strongly negative for the $\mathrm{NAO}^{+}$after lag +2 day. Thus, the downstream energy dispersion is more pronounced for the $\mathrm{NAO}^{+}$than for the $\mathrm{NAO}^{-}$during the NAO decaying phase. This difference can be further explained in terms of the indices defined below. We also tested the sensitivity of $D_{I}$ to the size of the average area for $H_{A}$ and $H_{D}$. It is found that the obtained results are held for other choices (not shown). It should be pointed out that there is a limitation that the $D_{I}$ index fails to capture the movement of wave trains related to the $\mathrm{NAO}$, though it can describe the energy dispersion of the NAO.

An indicator characterizing the dispersion of the $\mathrm{NAO}$ is to look at changes in the downstream blocking frequency before and after the NAO peak day. This is particularly apparent for the $\mathrm{NAO}^{+}$(Luo et al. 2007a). We calculate the time-mean blocking frequency (days) distributions before and after the peak day of the NAO and their difference by using the 2D-TM index and show the results in Fig. 5 for the $\mathrm{NAO}^{+}$and $\mathrm{NAO}^{-}$. Here, the time before (after) the NAO peak day is defined as a time interval from the beginning day of the NAO event to its peak day (the peak day of the NAO to its disappearance day).

It is found for the $\mathrm{NAO}^{+}$that blocking days are more frequent over Eurasia after than before the $\mathrm{NAO}^{+}$peak day (Figs. 5a and 5b). This can be clearly seen from their difference (Fig. 5c). This suggests that Eurasian blocking arises mainly from intensified downstream energy dispersion of the $\mathrm{NAO}^{+}$during its decay phase. In contrast, for the $\mathrm{NAO}^{-}$, blocking days are less frequent over Eurasia after the $\mathrm{NAO}^{-}$peak day (Fig. 5d) than before (Fig. 5e), probably because a large-scale trough occurs over Eurasia or the blocking itself shifts westward. The above results also suggest that the $\mathrm{NAO}^{+}$and $\mathrm{NAO}^{-}$ exhibit a strong asymmetry in their downstream circulation structures (Luo et al. 2007a), although there is a strong anticorrelation between blocking occurrence and the phase of the NAO (Croci-Maspoli et al. 2007). Below, we will further examine what causes the circulation asymmetry by exploring what factor influences the dispersion strength of the NAO. 

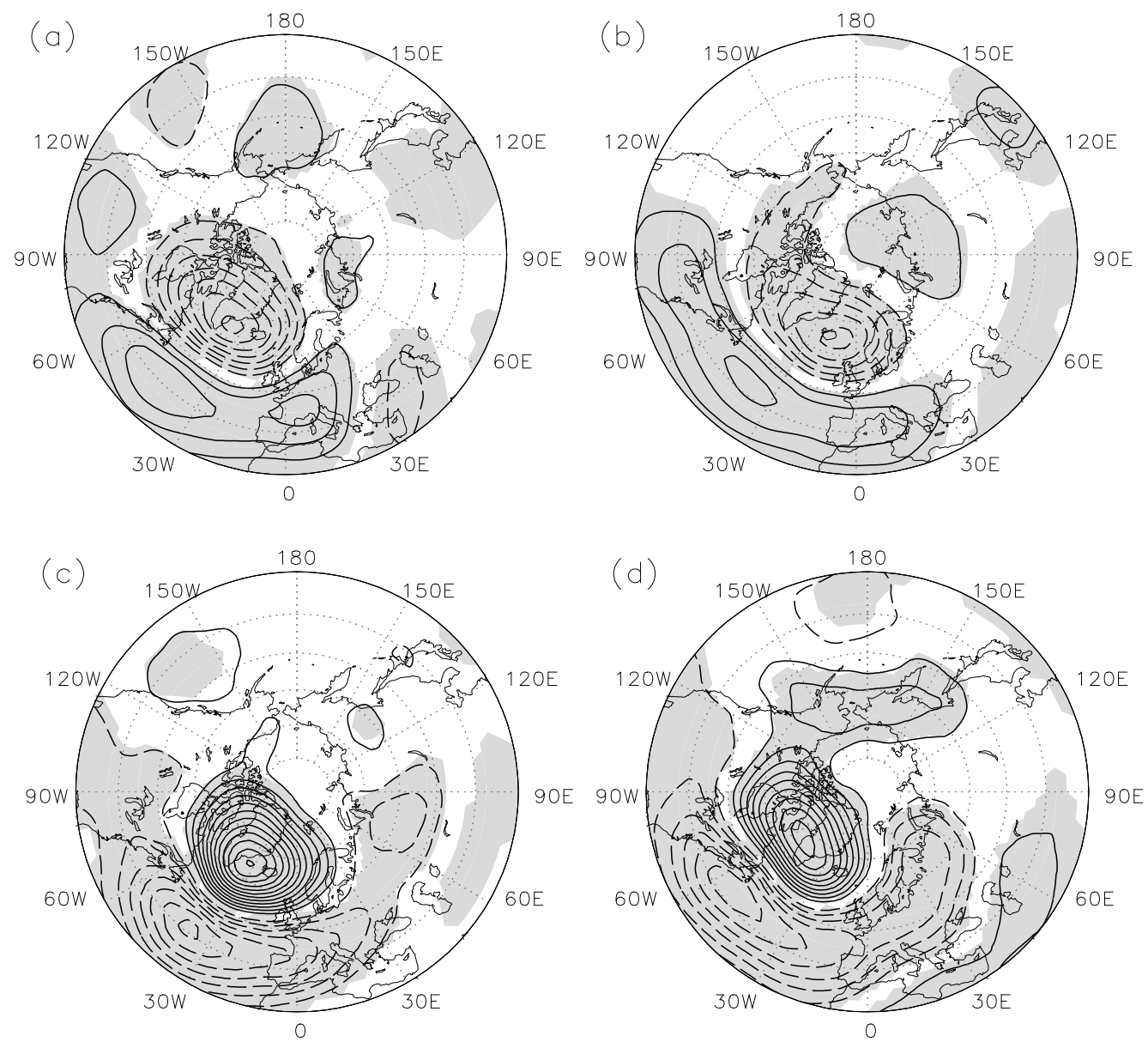

(e)

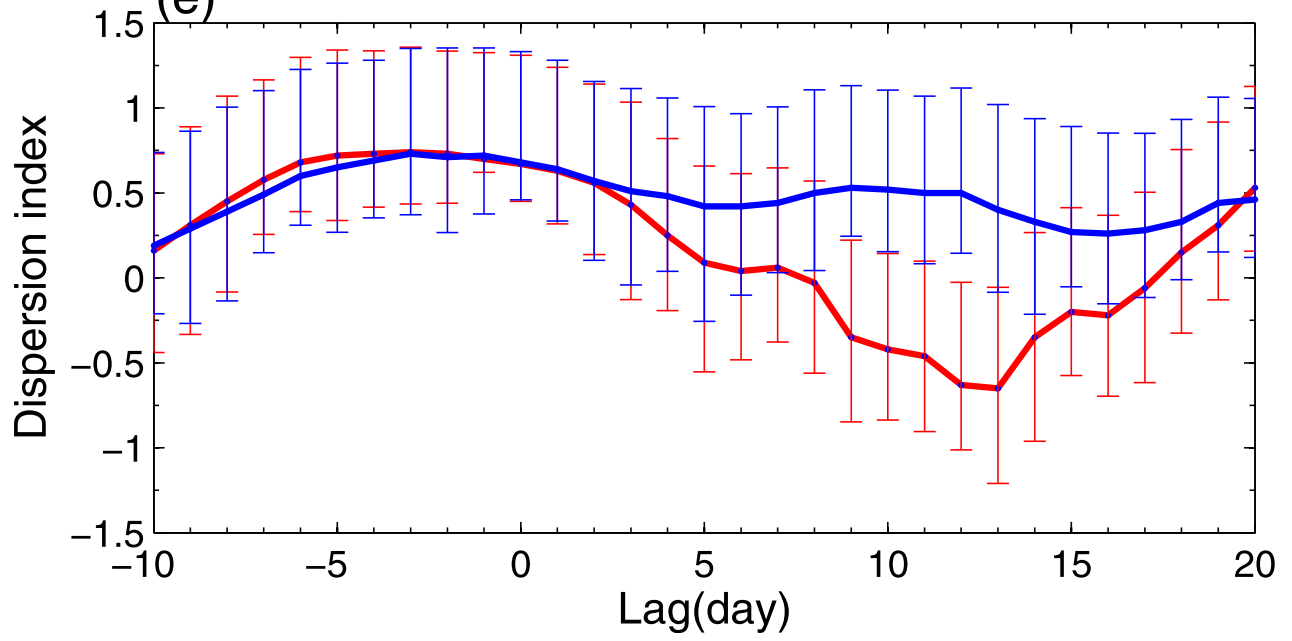

FIG. 4. Time-mean fields of composite daily 500-hPa geopotential height anomalies $(\mathrm{CI}=20 \mathrm{gpm})$ averaged from (a),(c) lag -5 to 0 days and (b),(d) lag 0 to 5 days for (a),(b) $\mathrm{NAO}^{+}$and (c),(d) $\mathrm{NAO}^{-}$events. (e) Time series of the dispersion index during the NAO life cycle for $\mathrm{NAO}^{+}$(red) and $\mathrm{NAO}^{-}$(blue) events during 1979-2015. Lag 0 denotes the day of the NAO peak. In (a)-(d), the gray shading denotes the regions above the $95 \%$ confidence level for the two-sided Student's $t$ test with a 5000-times simulation. In (e), the error bars indicate the upper 90th quartile and lower 10th quartile distribution 

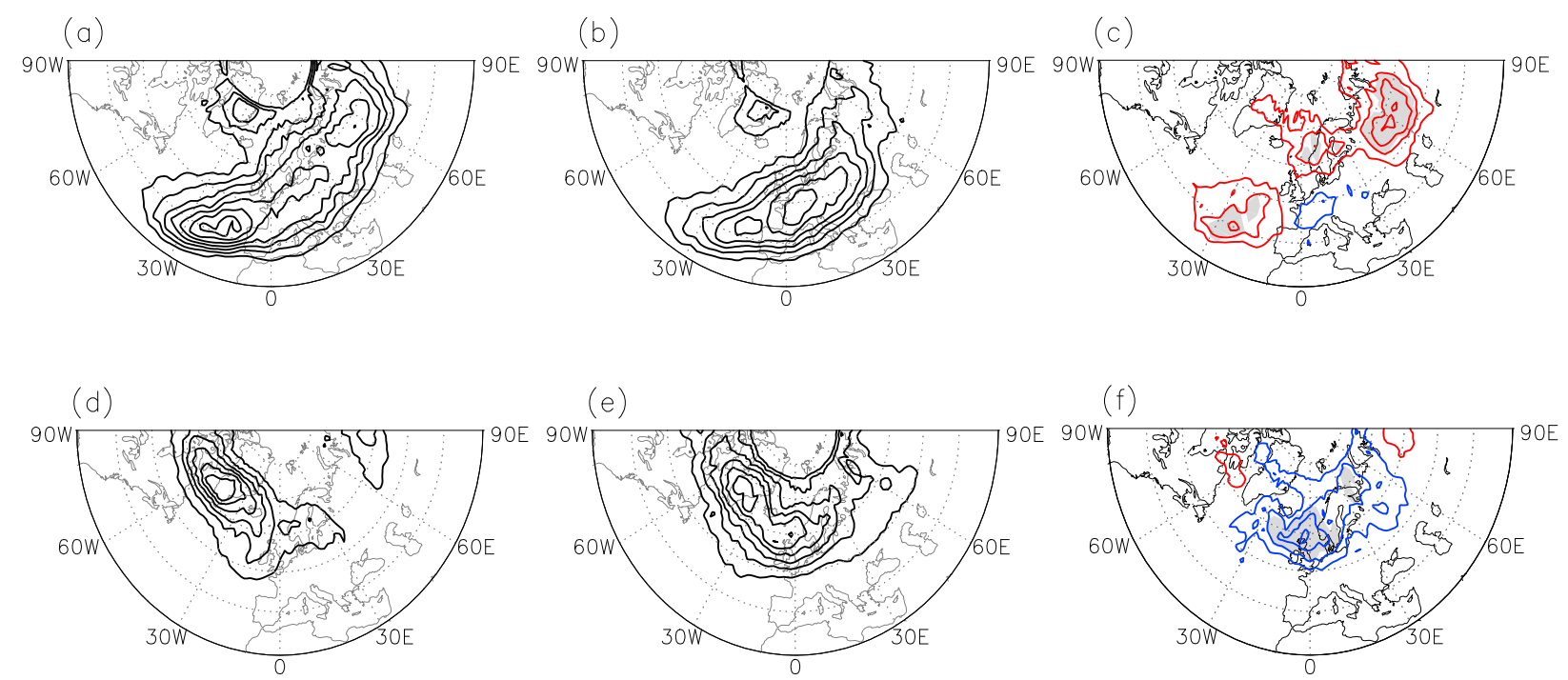

FIG. 5. Time-mean composite daily blocking frequency (contour interval $=0.3$ days) (a) after (from lag 0 to disappearance day) and (b) before (beginning day to lag 0) the $\mathrm{NAO}^{+}$peak day and (c) the (a) - (b) difference; (d) after and (e) before the $\mathrm{NAO}^{-}$peak day and (f) the (d) - (e) difference. The red (blue) shading denotes a positive (negative) blocking frequency anomaly. The gray shading in (c),(f) denotes the region above the $90 \%$ confidence level for a Monte Carlo test with a 5000-times simulation.

Another indicator of the $\mathrm{NAO}^{+}$dispersion is a large change in the longitudinal position of the Eurasian blocking from Europe to the Ural Mountains. Here, we define blocking events occurring in the domains $0^{\circ}-40^{\circ} \mathrm{E}$ and $40^{\circ}-80^{\circ} \mathrm{E}$ as the European blocking (EB) and Ural blocking (UB) events, respectively. The number of EB and UB events is calculated by using the 1D-TM index. It is found that there are $70 \mathrm{~EB}$ and $43 \mathrm{UB}$ events in winter during 1979-2015. We further define an $\mathrm{NAO}^{+}$-EB $\left(\mathrm{NAO}^{+}-\mathrm{UB}\right)$ event to have taken place if the peak of the EB (UB) event occurs over the life cycle of the $\mathrm{NAO}^{+}$ event. For a 1.0-STD definition of an $\mathrm{NAO}^{+}$event, there are $33 \mathrm{NAO}^{+}-\mathrm{EB}$ and $18 \mathrm{NAO}^{+}$-UB events during 1979-2015. We also find that blocking events related to the $\mathrm{NAO}^{-}$are infrequent (not shown). Here, we show the time mean of the composite daily $500-\mathrm{hPa}$ geopotential height anomalies averaged from lag -5 to 0 and lag 0 to 5 days for $\mathrm{NAO}^{+}-\mathrm{EB}$ or $\mathrm{NAO}^{+}-\mathrm{UB}$ events in Figs. $6 \mathrm{a}-\mathrm{d}$ (where lag 0 denotes the $\mathrm{NAO}^{+}$peak day), as well as the time variation of the dispersion index $D_{I}$ in Fig. 6e for $\mathrm{NAO}^{+}-\mathrm{EB}$ and $\mathrm{NAO}^{+}-\mathrm{UB}$ events.

It is found that the blocking intensity is distinctly different between the UB (Figs. 6c,d) and EB (Figs. 6a,b), which reflects a large difference in the dispersion strength of the $\mathrm{NAO}^{+}$between both cases. This result is not sensitive to the sample size of the NAO events (not shown). As further seen from Fig. 6e, the energy dispersion of the $\mathrm{NAO}^{+}$is stronger for the UB than for the EB. This is likely related to the stronger North Atlantic westerly wind for the $\mathrm{NAO}^{+}$-UB than that for the $\mathrm{NAO}^{+}$-EB
(Fig. 6f). Thus, it is inferred that the stronger North Atlantic westerly wind is easily followed by the excitation of the UB, whereas the EB requires a relatively weak North Atlantic westerly wind (red line in Fig. 6f). Thus, it is speculated that the strength of the North Atlantic westerly winds may also affect the dispersion of the $\mathrm{NAO}^{+}$. This will be further quantified in the next section.

As shown in Luo et al. (2007c), the North Atlantic westerly jet is distinctly different between the $\mathrm{NAO}^{-}$ and $\mathrm{NAO}^{+}$. In this case, it is natural to hypothesize that the strong asymmetry of the NAO is likely related to the large difference of the North Atlantic westerly winds between the $\mathrm{NAO}^{+}$and $\mathrm{NAO}^{-}$. To examine this problem, we show the time-latitude evolution of the composite daily zonal wind averaged over the region $50^{\circ} \mathrm{W}-0^{\circ}$ for $\mathrm{NAO}^{+}$and $\mathrm{NAO}^{-}$events in Fig. 7 . It is seen that the meridional position of the jet core of the North Atlantic jet is distinctly different between the $\mathrm{NAO}^{+}$and $\mathrm{NAO}^{-}$, which varies with the strength of the NAO (Figs. 7a,b) (Luo et al. 2007c; Strong and Davis 2008; Woollings et al. 2014). Especially, the jet core is located more northward for the $\mathrm{NAO}^{+}$than for the $\mathrm{NAO}^{-}$prior to the NAO onset (before lag -10 days) as the time-mean jet core from lag -20 to -10 days is located at $50^{\circ} \mathrm{N}\left(46^{\circ} \mathrm{N}\right)$ for the $\mathrm{NAO}^{+}$ $\left(\mathrm{NAO}^{-}\right)$. This difference is also evident in Fig. 7c. As mentioned by Luo et al. (2008b), the northward (southward) position of the preexisting North Atlantic jet may be considered as a precondition of the $\mathrm{NAO}^{+}\left(\mathrm{NAO}^{-}\right)$events. In fact, the meridional 

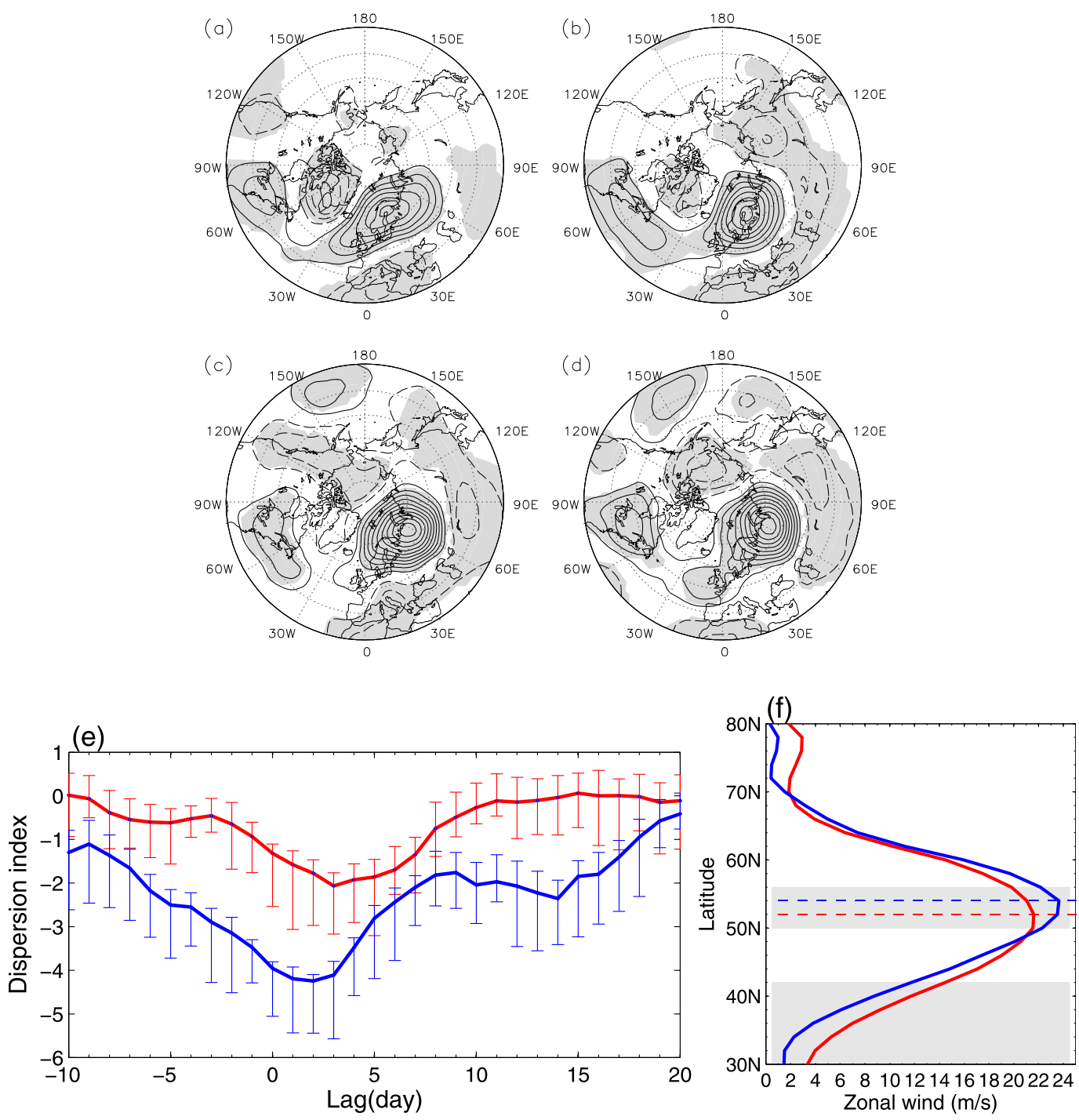

FIG. 6. Time-mean fields of composite daily 500-hPa geopotential height anomalies $(\mathrm{CI}=20 \mathrm{gpm})$ averaged from (a),(c) lag -5 to 0 days and (b),(d) lag 0 to 5 days for (a),(b) $\mathrm{NAO}^{+}-\mathrm{EB}$ and (c),(d) $\mathrm{NAO}^{+}-\mathrm{UB}$ events.(e) Time series of the daily dispersion index during the NAO life cycle for $\mathrm{NAO}^{+}$-EB (red) and $\mathrm{NAO}^{+}-\mathrm{UB}$ (blue) events, where lag 0 denotes the peak day of the blocking; (f) latitudinal profiles of time-mean composite daily westerly winds from lag -5 to +5 days and averaged over $50^{\circ} \mathrm{W}-0^{\circ}$ for $\mathrm{NAO}^{+}$-EB (red) and $\mathrm{NAO}^{+}$-UB events, where lag 0 denotes the $\mathrm{NAO}^{+}$peak. The gray shading denotes the region above the $95 \%$ confidence level for the two-sided Student's $t$ test in (a)-(d) but for a Monte Carlo test with a 5000-times simulation in (f). In (e), the error bars indicate the upper 90th quartile and lower 10th quartile distribution.

position of the preexisting North Atlantic jet is more likely dominated by the sea surface temperature (SST) in the Gulf Stream (O'Reilly et al. 2017) rather than by the synoptic-scale eddy forcing before the NAO onset because the eddy forcing is extremely weak before lag -10 days (Barnes and Hartmann 2010).

As the NAO grows, the North Atlantic jet is further intensified (weakened) for the $\mathrm{NAO}^{+}\left(\mathrm{NAO}^{-}\right)$, and its jet core undergoes a northward (southward) shift from its mean position (Fig. 7d). We can see in Fig. 7d that during the NAO mature phase (from lag -5 to 5 days), the time-mean jet core shifts to $52^{\circ}\left(36^{\circ}\right) \mathrm{N}$ from its initial position at $50^{\circ}\left(46^{\circ}\right) \mathrm{N}$ for the $\mathrm{NAO}^{+}\left(\mathrm{NAO}^{-}\right)$. It is interesting to find that the $\mathrm{NAO}^{-}\left(\mathrm{NAO}^{+}\right)$corresponds to a large (small) southward (northward) shift of the North Atlantic jet core. The NAO alone cannot satisfactorily explain the variability in speed and latitude of the North Atlantic jet (Woollings et al. 2010), probably because the North Atlantic jet describes a more complex spatial pattern of the large-scale circulation than that of 
(a)

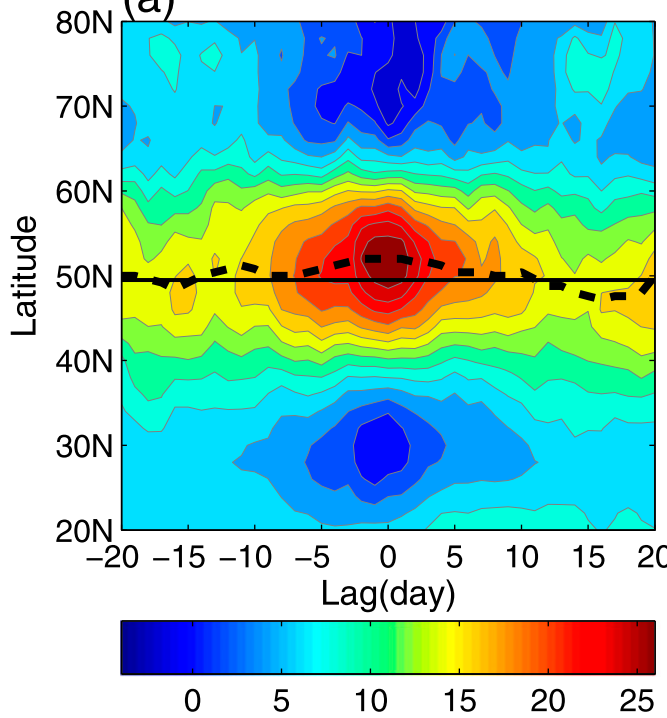

(c)

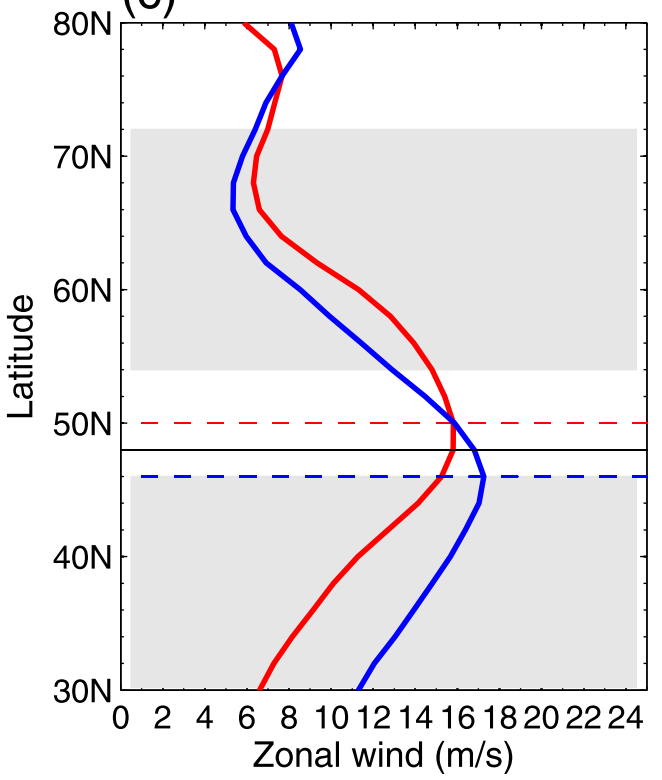

(b)

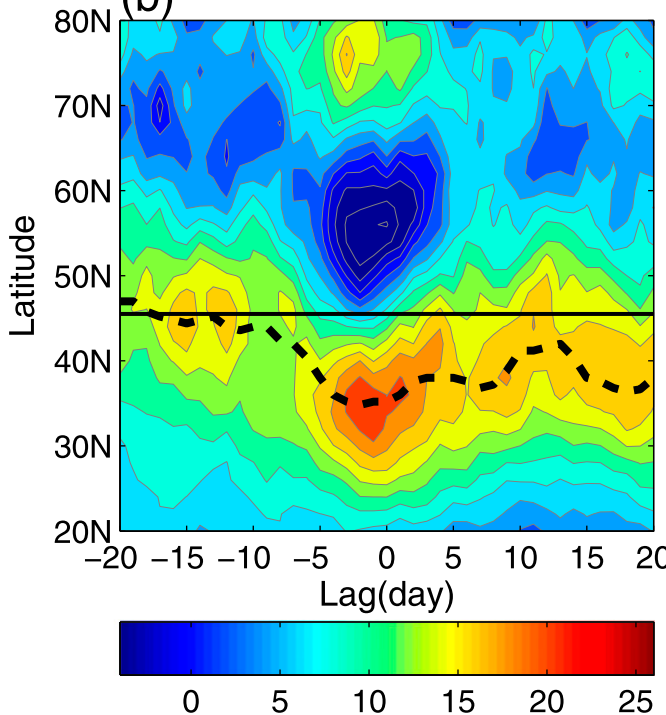

(d)

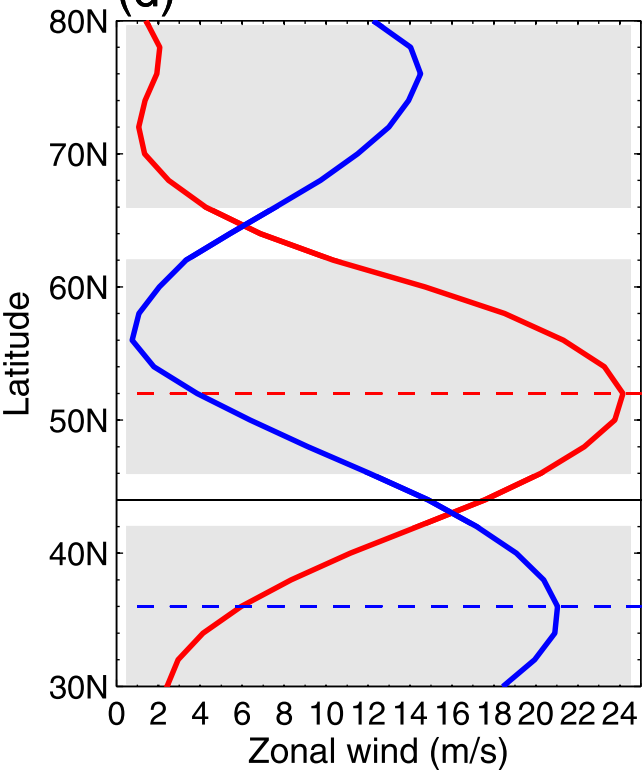

FIG. 7. (a),(b) Latitude-time sections of composite daily zonal winds $\left(\mathrm{m} \mathrm{s}^{-1}\right)$ averaged over the region $50^{\circ} \mathrm{W}-0^{\circ}$ for $\mathrm{NAO}^{+}$and $\mathrm{NAO}^{-}$events during 1979-2015, respectively, in which the solid line represents the time-mean value from lag -20 to -10 days. (c),(d) The variation of time-mean zonal-averaged zonal winds (c) from lag -20 to -0 days and (d) from lag -5 to +5 days with the latitude for $\mathrm{NAO}^{+}$(red line) and $\mathrm{NAO}^{-}$ (blue line) events, where the solid line represents the mean position of the North Atlantic jet for its two phases. The red (blue) dashed line represents the maximum position of the composite North Atlantic jet for the $\mathrm{NAO}^{+}\left(\mathrm{NAO}^{-}\right)$events. In (c) and (d), the gray shading denotes the $95 \%$ confidence level for a Monte Carlo test with a 5000-times simulation.

the NAO alone, and it is modulated by the North Atlantic SST (O'Reilly et al. 2017). But the phase of the NAO pattern can in large part explain the variability of the North Atlantic jet on a 10-20-day time scale (Luo et al. 2007c). Moreover, it is seen that the maximum strength of the time-mean North Atlantic westerly wind changes from $15.8(17.2) \mathrm{m} \mathrm{s}^{-1}$ during the prior phase (from lag -20 to -10 days) of the NAO to 24.2 (21.0) $\mathrm{m} \mathrm{s}^{-1}$ during its mature phase for the $\mathrm{NAO}^{+}$ $\left(\mathrm{NAO}^{-}\right)$as the NAO intensifies. Thus, the strength and position of the North Atlantic westerly wind show a strong asymmetry between the $\mathrm{NAO}^{+}$and $\mathrm{NAO}^{-}$. Below, we 
will indicate that the asymmetry of the North Atlantic westerly jet is the main cause of the symmetry breaking of the NAO between its two phases.

\section{Asymmetries of the dispersion and nonlinearity of the NAO and the basic physics of symmetry breaking}

\section{a. Definitions of dispersion and nonlinearity indices}

Although some studies attempted to explain the asymmetry of the NAO persistence (Barnes and Hartmann 2010), no complete theory was proposed to account for the asymmetries of the NAO in zonal movement, amplitude, persistence, and flow pattern. Motivated by the UNMI model, here we present new indices to account for why the NAO shows strong asymmetry in amplitude, persistence, and zonal movement between its two phases. The definition of these indices is based on the evolution equation of the NAO amplitude described by Eq. (3h). Here, we do not present the theoretical result of the UNMI model because it has been previously used to examine the dynamics of blocking and NAO events (Luo 2000, 2005; Luo et al. 2007a,b,c, 2014, 2015). Instead, we only examine the physical cause of the NAO asymmetry from a reanalysis data perspective.

However, it must be pointed out that the derivation of Eq. (3h) for the NAO amplitude is based on an important assumption that the mean zonal wind is fixed as a constant. In fact, the mean zonal wind changes in time and space and are coupled together with the NAO anomaly once the NAO occurs (Luo et al. 2007c). In this case, $C_{g}, \lambda, \beta+F u_{0}$, and $\delta$ in Eq. (3h) change in space and time, and their corresponding mean zonal wind should be replaced by the local zonal wind (as defined below) because $C_{g}, \lambda, \beta+F u_{0}$, and $\delta$ change with the strength and spatial distribution of the local zonal wind.

The solution (3d) shows that the mean zonal wind will change once an NAO occurs. For this case, the mean westerly wind is $u_{M}=u_{0}-\partial \psi_{m} / \partial y$, which is proportional to the NAO amplitude squared $|B|^{2}$ For this case, the local zonal wind of the NAO system can be written as $u=u_{M}+$ $u_{\mathrm{NAO}}$, where $u_{\mathrm{NAO}}=-\partial \psi_{\mathrm{NAO}} / \partial y$ is the geostrophic zonal wind of the NAO and is proportional to the NAO amplitude $B$ in Eq. (3d). It is clear that we are also able to consider the effect of the zonal wind change on the NAO pattern if $u_{0}$ in $C_{g}, \lambda, \beta+F u_{0}$, and $\delta$ in Eq. (3h) is replaced by the local zonal wind $u$. Moreover, $\beta$ in all the mathematical expressions should be replaced by $\beta^{*}=Q_{y}=\beta+q_{y}$ in our real calculation, where $Q=\beta y+q$ and $q=\nabla^{2} \psi$ are the absolute and relative vorticity, respectively, and the subscript $y$ denotes the meridional gradient. This allows us to examine how changes in the dispersion and nonlinearity related to the zonal wind change affect the evolution of the NAO and its asymmetry.

We define the difference between the group velocity and phase speed $C_{\mathrm{gp}}=C_{g}-C_{p}$ as a dispersion index, which measures the linear energy dispersion of the NAO system, where $C_{p}=\omega / k$ is the phase speed. The NAO system is nondispersive if $C_{\mathrm{gp}} \approx 0$. But it becomes dispersive if $C_{\mathrm{gp}} \neq 0$. As noted below, $C_{\mathrm{gp}}, C_{g}$, and $\lambda$ have consistent variations. Thus, our emphasis below is placed on the variation of $C_{\mathrm{gp}}$, though the results of $C_{g}$ and $\lambda$ are also given. In particular, when $C_{\mathrm{gp}}$ and $\lambda$ are larger and when $\delta$ is smaller in Eq. (3h), the NAO tends to be a linear process because there is $i\left(\partial B / \partial t+C_{g} \partial B / \partial x\right)+\lambda \partial^{2}$ $B / \partial^{2} x \approx 0$ in the absence of forcing. In contrast, the NAO pattern tends to be a strongly nonlinear process. This strongly nonlinear process implies that a large-amplitude and long-lived NAO easily forms because of the vanishing of strong energy dispersion.

In terms of the asymptotic solution to Eq. (3h), the nonlinear phase speed $C_{\mathrm{NP}}$ of the NAO anomaly can be approximately obtained as $C_{\mathrm{NP}}=u-\left(\beta^{*}+F u\right)\left(k^{2}+m^{2}+F\right)-$ $\delta M_{0}^{2} /(2 k)$ (Luo 2000; Luo et al. 2011), where $u$ is the local zonal wind, $\beta$ has been replaced by the local $\beta^{*}$ as defined above, and $M_{0}=|B|_{\max }$ denotes the maximum amplitude per day. The value of $C_{\mathrm{NP}}$ reflects the zonal movement of the NAO anomaly per latitude. When $C_{\mathrm{NP}}>0\left(C_{\mathrm{NP}}<0\right)$, the NAO shifts eastward (westward).

We also define $V=\delta|B|^{2}$ in Eq. (3h) to describe the strength of the nonlinearity even though $\delta$ measures the nonlinearity in Eq. (3h). In fact, $V=\delta|B|^{2}$ reflects the combination of the nonlinearity $\delta$ and the amplitude effect of the NAO because the large amplitude often corresponds to strongly nonlinear behavior. In this case, Eq. (3h) can be rewritten in the form of $i(\partial B / \partial t+$ $\left.C_{g} \partial B / \partial x\right)+\lambda \partial^{2} B / \partial^{2} x+V(B) B+G f_{0}^{2} \exp [-i(\Delta k x+$ $\Delta \omega t)]=0$. Here, we refer to $V=\delta|B|^{2}$ as the nonlinearity index of the NAO anomaly. It should be mentioned that $V=\delta|B|^{2}$ depends not only on the magnitude of the nonlinear coefficient $\delta$ but also on the amplitude $|B|$ of the NAO anomaly. When $V=$ $\delta|B|^{2}$ is larger, Eq. (3h) becomes a strongly nonlinear equation. But when $V=\delta|B|^{2}$ is smaller, Eq. (3h) tends to be a linear equation with a forcing term. To some extent, the magnitudes of $C_{\mathrm{gp}}$ and $V=\delta|B|^{2}$ reflect linear and nonlinear behavior of the NAO pattern. This will be quantified in the following subsections. In our calculation below, we choose the parameters $L=5$ $\left(5000 \mathrm{~km}\right.$ in a dimensional form) and $\phi_{0}=55^{\circ} \mathrm{N}$. Here, we further define $\tilde{M}_{0}$ as the maximum amplitude of the NAO height anomaly $\psi_{\text {NAO }}$ per latitude. For this case, the maximum amplitude $M_{0}$ of the NAO anomaly should be $\tilde{M}_{0}$ divided by $2\left(2 / L_{y}\right)^{1 / 2}$ in that 
(a)

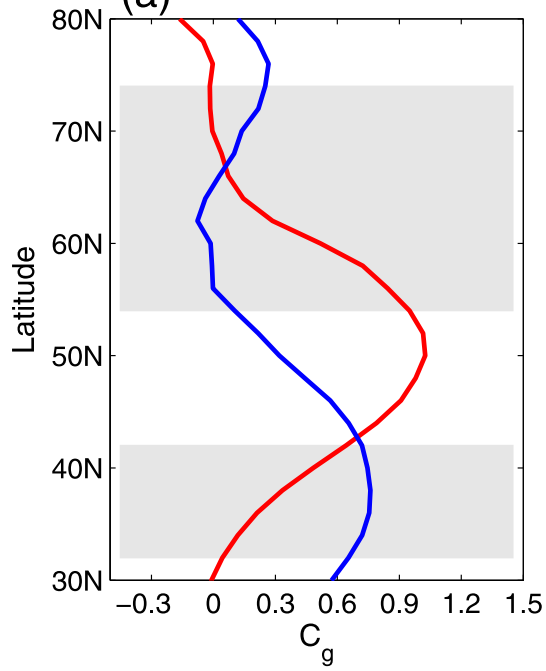

(d)

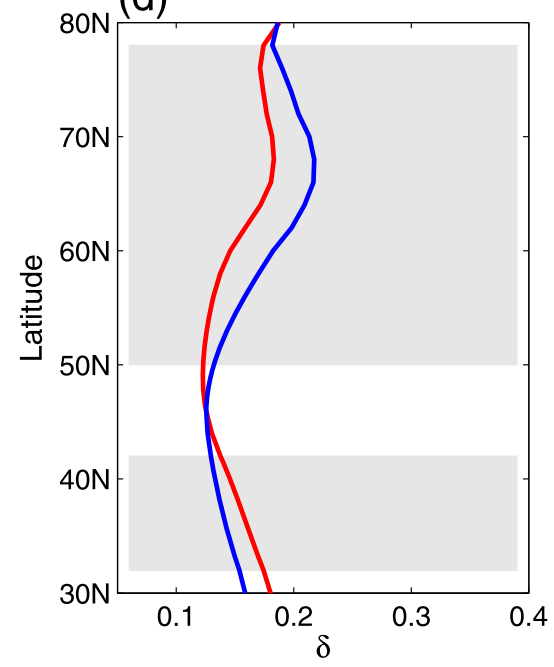

(b)

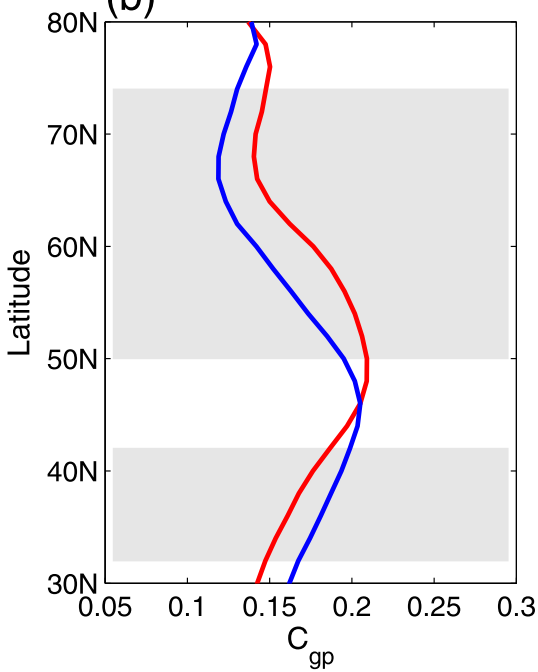

(e)

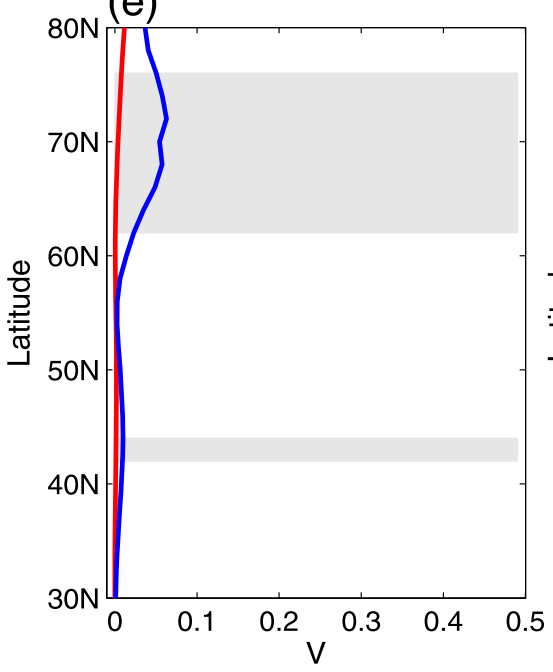

(c)

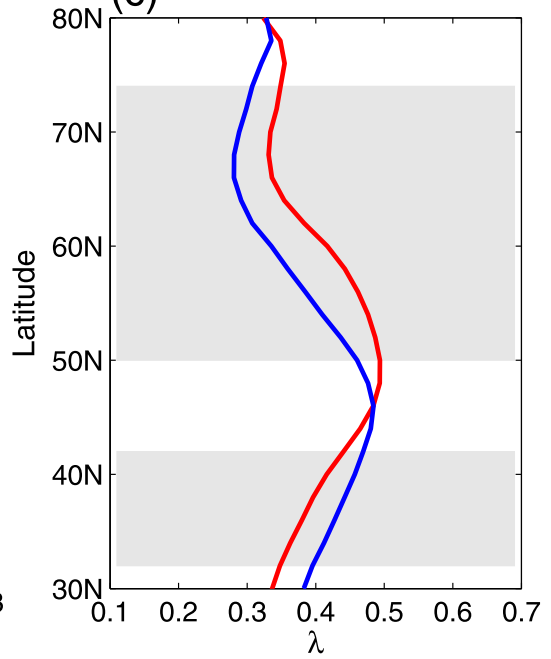

(f)

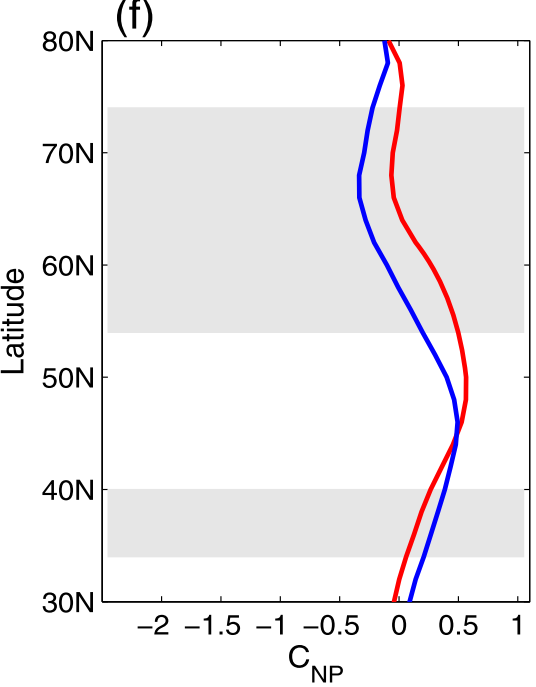

FIG. 8. The distributions of time-mean nondimensional (a) $C_{g}$, (b) $C_{\mathrm{gp}}$, (c) $\lambda$, (d) $\delta$, (e) $V$, and (f) $C_{\mathrm{NP}}$ averaged over the region $50^{\circ} \mathrm{W}-0^{\circ}$ from lag -20 to -10 days with the latitude for the $\mathrm{NAO}^{+}$(red) and $\mathrm{NAO}^{-}$(blue) events during 1979-2015. The gray shading denotes the $95 \%$ confidence level for a Monte Carlo test with a 5000-times simulation.

$\psi_{\mathrm{NAO}}=2 \operatorname{Re}\left\{B\left(2 / L_{y}\right)^{1 / 2} \exp [i(k x-\omega t)] \sin (m y)\right\}$ where Re denotes the real part.

\section{b. Dispersion and nonlinearity changes during the $N A O$ life cycle and their link to the asymmetry of the NAO pattern}

Before examining the above problem, we first show the time mean of the composite daily $C_{g}, C_{\mathrm{gp}}, \lambda, \delta, V$, and $C_{\mathrm{NP}}$ averaged over the region $50^{\circ} \mathrm{W}-0^{\circ}$ and over the time interval from lag -20 to -10 days (prior to the NAO onset) in Fig. 8. The values of these variables may be considered as the background condition of the NAO onset. It is seen that $C_{g}, C_{\mathrm{gp}}$, and $\lambda(\delta$ and $V$ ) prior to the NAO onset are smaller (larger) for the $\mathrm{NAO}^{-}$than for the $\mathrm{NAO}^{+}$(Fig. 8). Thus, it is concluded that an $\mathrm{NAO}^{-}$anomaly with a larger amplitude and longer persistence is likely due to a weaker prior North Atlantic westerly wind in high latitudes (Fig. 7c). However, the difference of $C_{g}, C_{\mathrm{gp}}, \lambda, \delta, V$, and $C_{\mathrm{NP}}$ becomes more significant once the $\mathrm{NAO}^{-}\left(\mathrm{NAO}^{+}\right)$ occurs and strengthens, because the North Atlantic westerly wind is further weakened (intensified) (Fig. 7d). This problem will be further quantified below.

To establish a physical link between the asymmetries of the dispersion and nonlinearity of the NAO and westerly wind changes, we show the time-latitude variation of the composite daily $C_{g}, C_{\mathrm{gp}}, \lambda, \delta, V$, and $C_{\mathrm{NP}}$ averaged over the region $\left(50^{\circ} \mathrm{W}-0^{\circ}\right)$ during the NAO life cycle in Figs. 9 and 10 for the $\mathrm{NAO}^{+}$and $\mathrm{NAO}^{-}$, respectively. It is seen that while $C_{g}, C_{\mathrm{gp}}$, and $\lambda$ increase in the mid- to high-latitude region (Figs. 9a-c) because of 
(a)

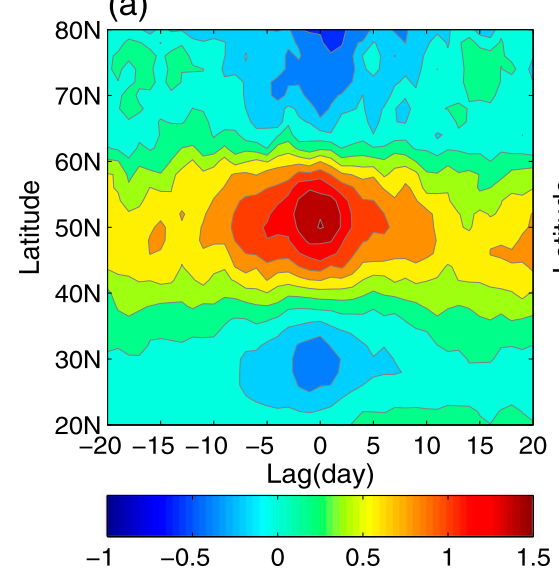

(d)

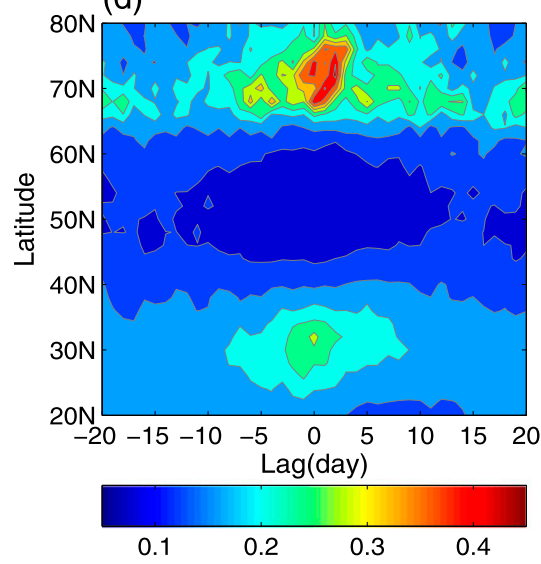

(b)

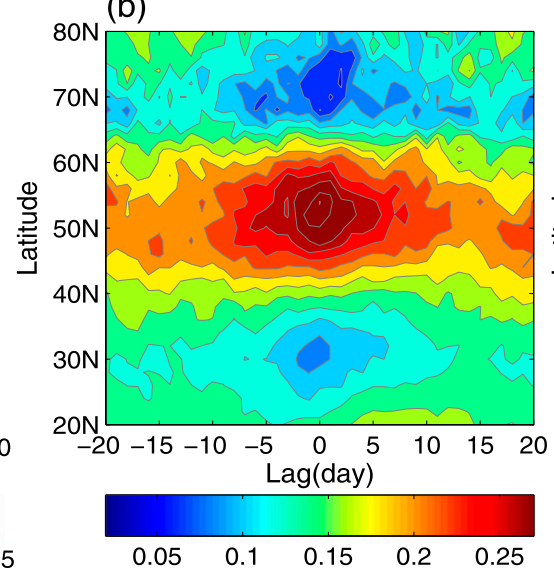

(e)

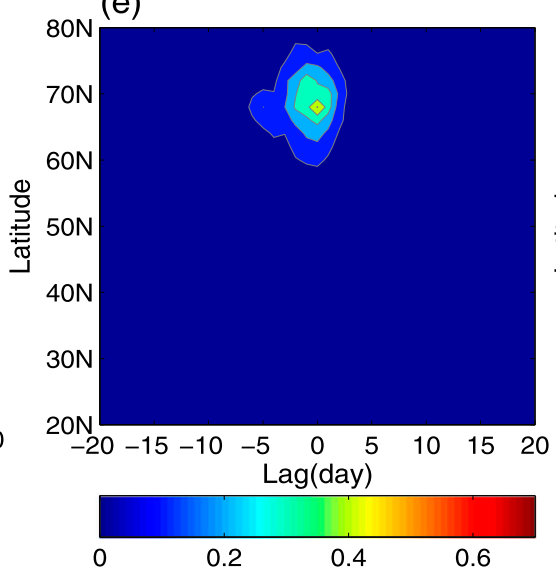

(c)
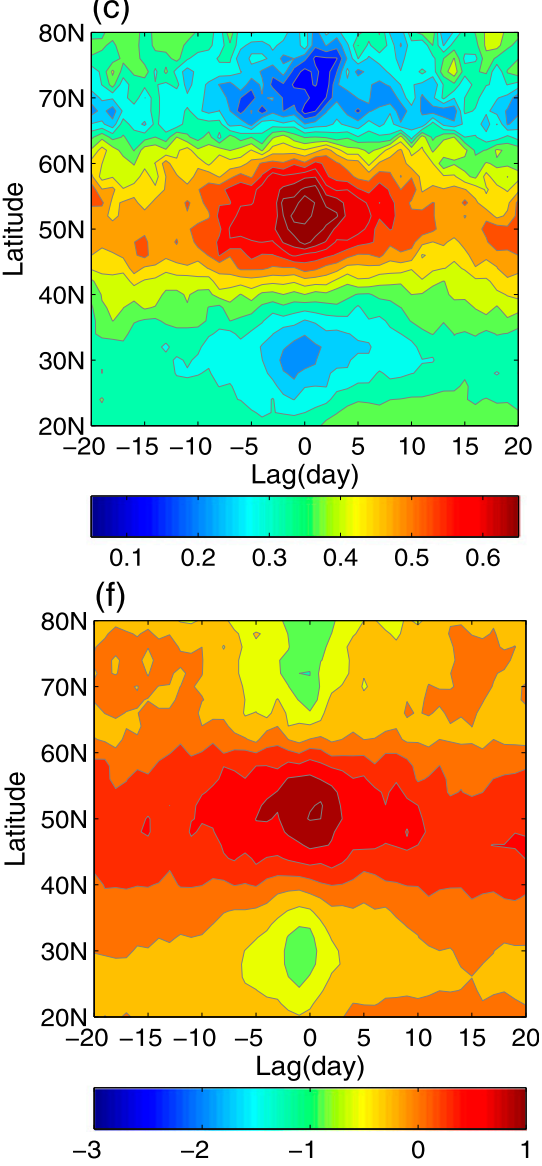

FIG. 9. Latitude-time evolution of composite daily nondimensional (a) $C_{g}$, (b) $C_{\mathrm{gp}}$, (c) $\lambda$, (d) $\delta$, (e) $V$, and (f) $C_{\mathrm{NP}}$ averaged over the region $50^{\circ} \mathrm{W}-0^{\circ}$ for the $\mathrm{NAO}^{+}$events during $1979-2015$.

the strengthening and northward shifting of the North Atlantic jet during the $\mathrm{NAO}^{+}$life cycle (Fig. 7a), both $\delta$ and $V$ decrease (Figs. 9d,e). This situation corresponds to enhanced downstream energy dispersion and a reduced nonlinearity of the $\mathrm{NAO}^{+}$, thus suggesting that the $\mathrm{NAO}^{+}$ tends to be a linear process. On the other hand, we see that $C_{\mathrm{NP}}$ is also significantly increased (Fig. 9f) as the North Atlantic westerly wind is intensified for the $\mathrm{NAO}^{+}$(Fig. 7a). This implies that the $\mathrm{NAO}^{+}$shows a pronounced eastward displacement during its life cycle. These results may explain why the observed $\mathrm{NAO}^{+}$has less persistence, a smaller amplitude, and eastward movement.

As we find from Fig. 10, $C_{g}, C_{\mathrm{gp}}, \lambda$, and $C_{\mathrm{NP}}$ decrease with the growth of the $\mathrm{NAO}^{-}$in the mid- to high-latitude region (Figs. 10a-c and Fig. 10f), while $\delta$ and $V$ increase (Figs. 10d,e). These parameters show opposite variation to those of the $\mathrm{NAO}^{+}$. Thus, the growth of the $\mathrm{NAO}^{-}$ essentially corresponds to a weakened energy dispersion and enhanced nonlinearity because the North Atlantic jet is weakened and shifted southward (Figs. $7 \mathrm{~b}$ and $7 \mathrm{~d}$ ). This allows a large-amplitude and long-lived $\mathrm{NAO}^{-}$to easily form (Fig. 3), thus indicating that the $\mathrm{NAO}^{-}$tends to be a strongly nonlinear process. Moreover, the $\mathrm{NAO}^{-}$is seen to undergo a pronounced westward movement because $C_{\mathrm{NP}}$ is largely negative during its life cycle.

To see whether the changes in $C_{g}, C_{\mathrm{gp}}, \lambda, \delta, V$, and $C_{\mathrm{NP}}$ between the $\mathrm{NAO}^{-}$and $\mathrm{NAO}^{+}$are significant, it is useful to show the meridional distribution of the time-mean $C_{g}, C_{\mathrm{gp}}, \lambda, \delta, V$, and $C_{\mathrm{NP}}$ averaged over $50^{\circ} \mathrm{W}-0^{\circ}$ and from lag -5 to 5 days (mature period) for the $\mathrm{NAO}^{-}$ and $\mathrm{NAO}^{+}$(Fig. 11). It is interesting to see that the differences of these parameters between the $\mathrm{NAO}^{-}$ (blue line in Fig. 11) and $\mathrm{NAO}^{+}$(red line in Fig. 11) are statistically significant at the $95 \%$ confidence level for a Monte Carlo simulation. In other words, the symmetry breaking or asymmetry of the NAO between its two phases is closely related to their significant changes in the dispersion and nonlinearity of the NAO between its two phases because of large changes in the North Atlantic westerly winds. Thus, it is concluded that the symmetry breaking of the NAO is a natural outcome of the NAO occurrence. 
(a)

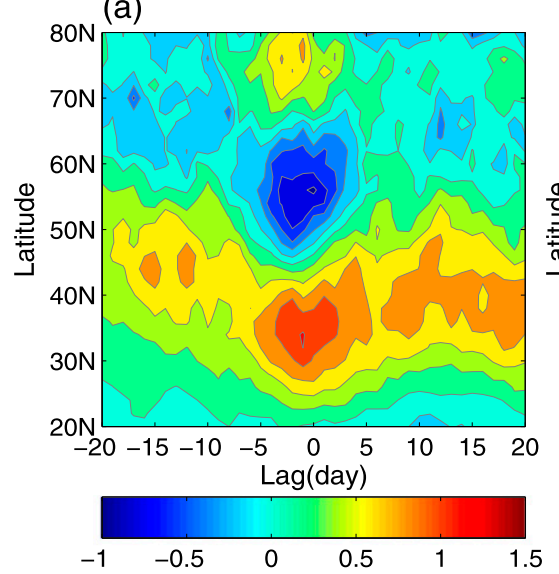

(d)

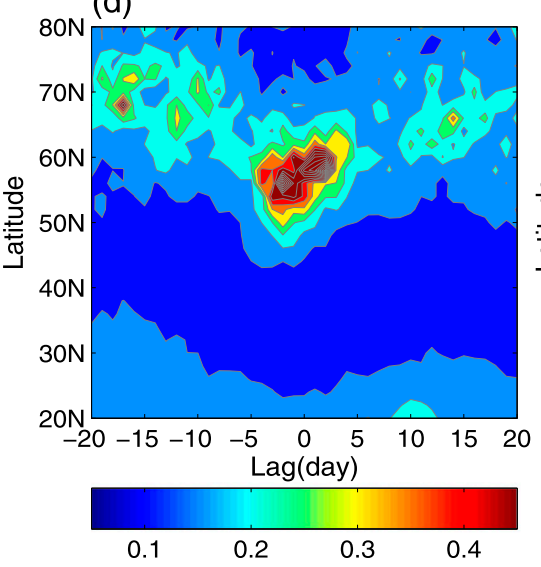

(b)

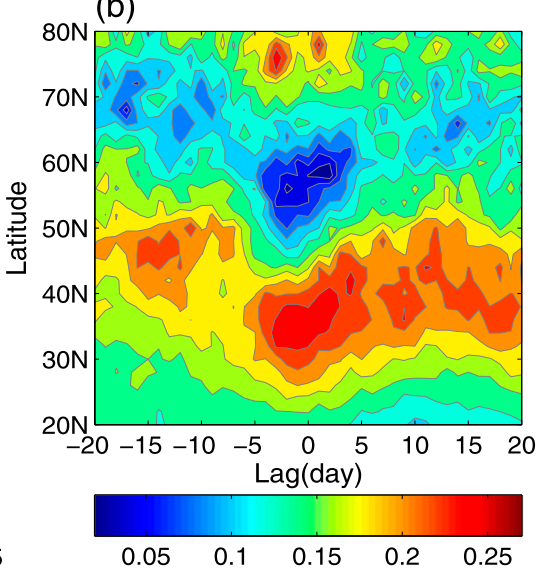

(e)

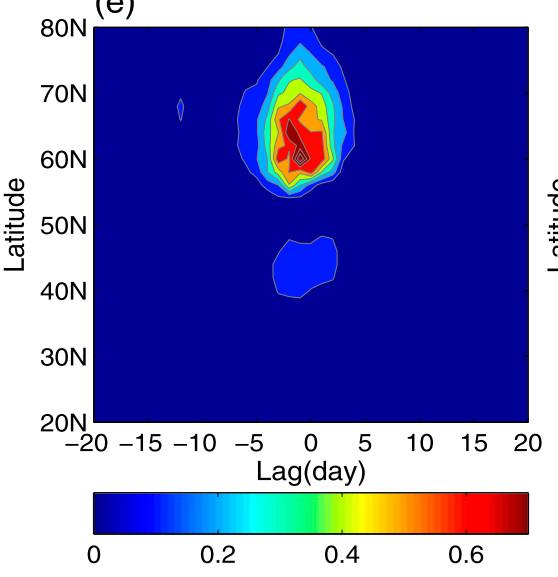

(c)

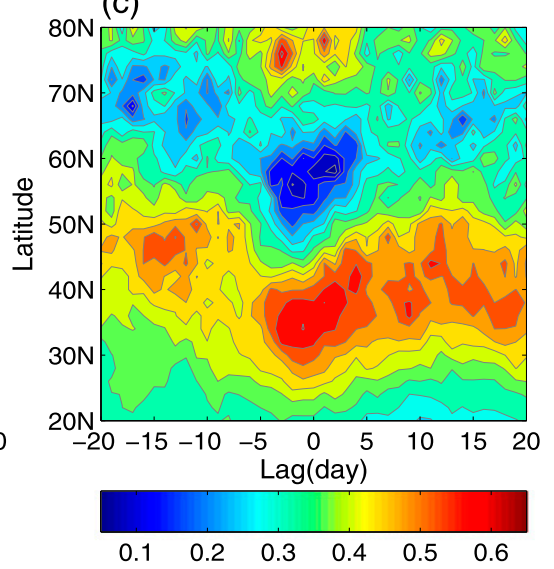

(f)

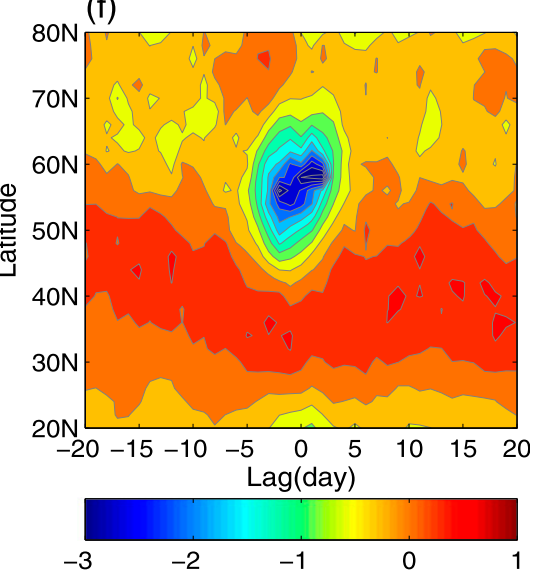

FIG. 10. As in Fig. 9, but for $\mathrm{NAO}^{-}$events during 1979-2015.

To isolate the different influences of the $\beta$ variation and large-scale wind on the NAO, we also consider two cases: 1) $\beta$ is varied with the latitude, and the zonal wind is a constant and 2) $\beta$ is a constant, and the local zonal wind is changed. Here, we first examine the dependence of $C_{\mathrm{gp}}$ and $\delta$ on latitude by assuming that the zonal wind is a constant and $\beta$ changes with latitude. It can be shown that $\delta$ is larger in higher latitudes than in lower latitudes, while $C_{\mathrm{gp}}$ is smaller in higher latitudes for the $\mathrm{NAO}^{-}$than for the $\mathrm{NAO}^{+}$ (not shown). This indicates that the $\mathrm{NAO}^{-}$more likely becomes weakly dispersive or even nondispersive in higher latitudes than the $\mathrm{NAO}^{+}$. To some extent, this can explain why the northern node of the $\mathrm{NAO}^{-}$has larger amplitude and longer persistence. Moreover, we find that even for a constant $\beta$, $\delta$ is strong (weak), and $C_{\mathrm{gp}}$ is small (large) because of the weakened (intensified) large-scale zonal wind related to the $\mathrm{NAO}^{-}\left(\mathrm{NAO}^{+}\right)$(not shown). Thus, we infer that the $\mathrm{NAO}^{-}\left(\mathrm{NAO}^{+}\right)$becomes a nonlinear (linear) system because of the latitude effect and the large-scale wind changes within the NAO itself.
To help understand whether the dependence of NAO dispersion on the amplitude of the NAO is distinctly different between its two phases, it is useful to first define the nondimensional amplitude of the NAO anomaly in the form of $A_{m}=\alpha_{\Re} Z_{m}$, where $\alpha_{\Re}=g /\left(f U_{0} L\right) \approx 1 / 100$ for $g \sim 9.8 \mathrm{~m} \mathrm{~s}^{-2}, f \sim 10^{-4} \mathrm{~s}^{-1}, L=10^{6} \mathrm{~m}, R_{d}=10^{6} \mathrm{~m}$, $U_{0}=10 \mathrm{~m} \mathrm{~s}^{-1}$, and $Z_{m}$ is the maximum value at the cyclonic (anticyclonic) center for the $\mathrm{NAO}^{+}\left(\mathrm{NAO}^{-}\right)$ events. We show the scatterplot of the time-mean (from lag -5 to 5 days) nondimensional NAO amplitude against the time-mean, domain-averaged $C_{\mathrm{gp}}$ and zonal wind $u$ (Fig. 12) for the two phases of the NAO, where the robust-fit function has been used to obtain the linear bestfit line. It can be seen that the dispersion strengthens as the amplitude of the $\mathrm{NAO}^{+}$increases (Fig. 12a), because the large-amplitude $\mathrm{NAO}^{+}$corresponds to a strong zonal wind (Fig. 12c). This indicates that the larger the $\mathrm{NAO}^{+}$ amplitude, the stronger its energy dispersion. In contrast, the dispersion of the $\mathrm{NAO}^{-}$is weakened as its amplitude is increased (Fig. 12b) because the zonal wind decreases with the increase of the $\mathrm{NAO}^{-}$amplitude (Fig. 12d). Consequently, it is concluded from the above results that a 
(a)

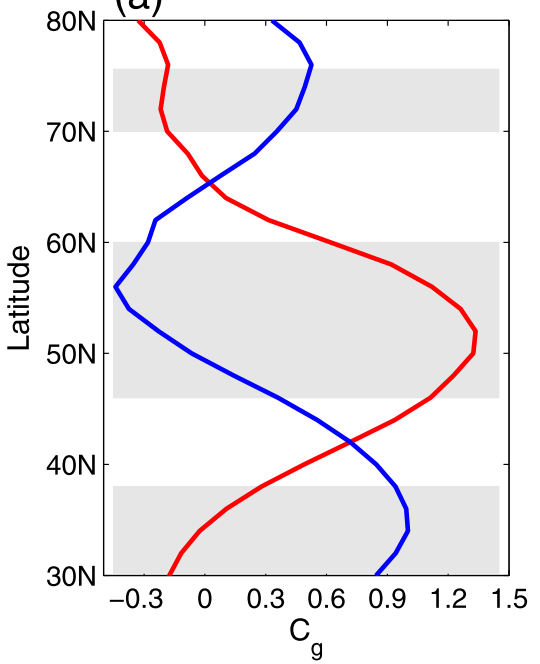

(d)

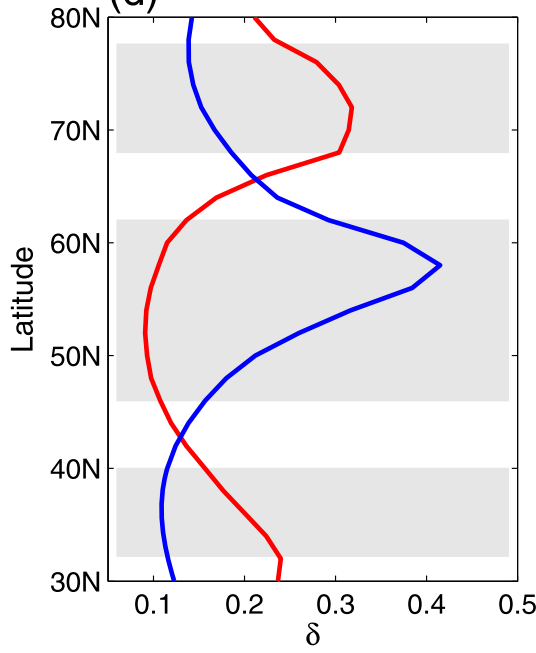

(b)

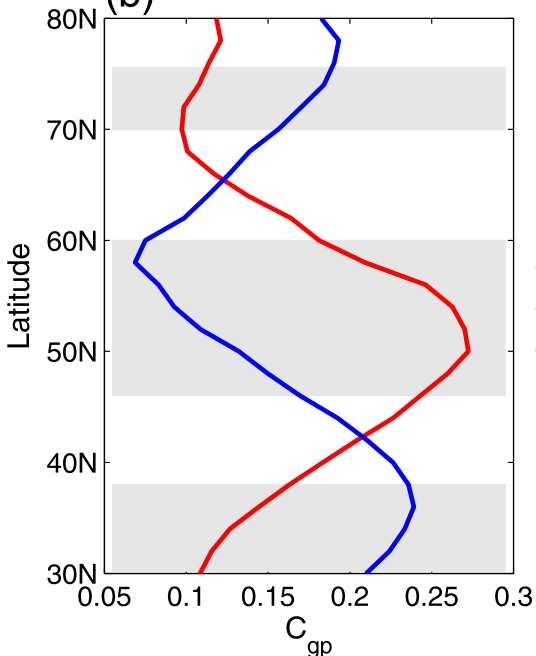

(e)

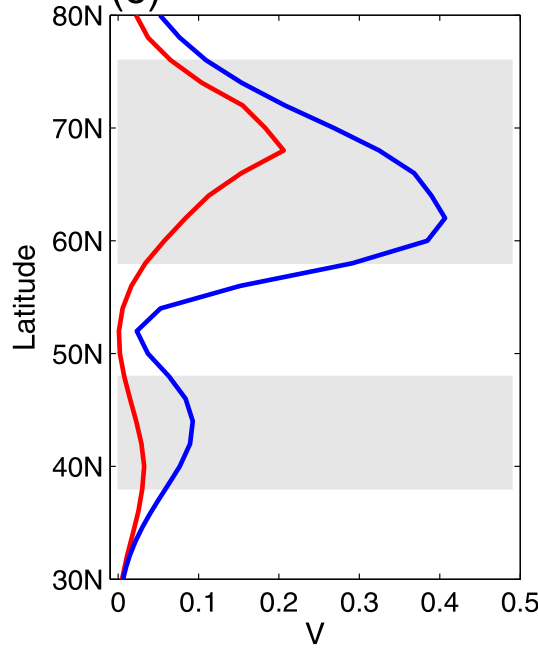

(c)

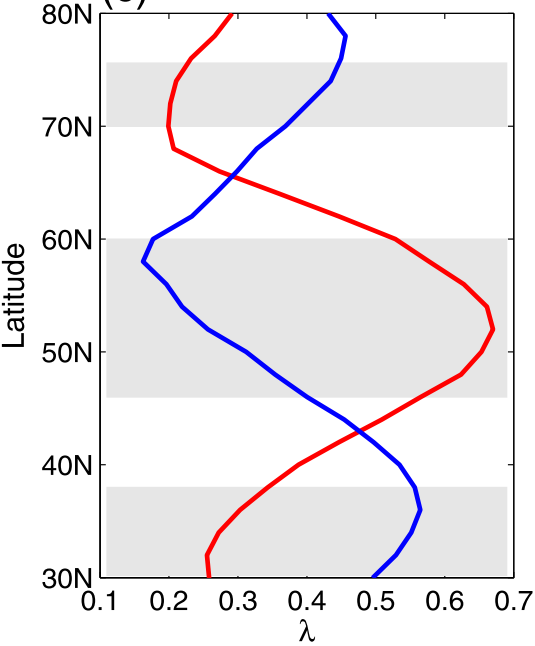

$(\mathrm{f})$

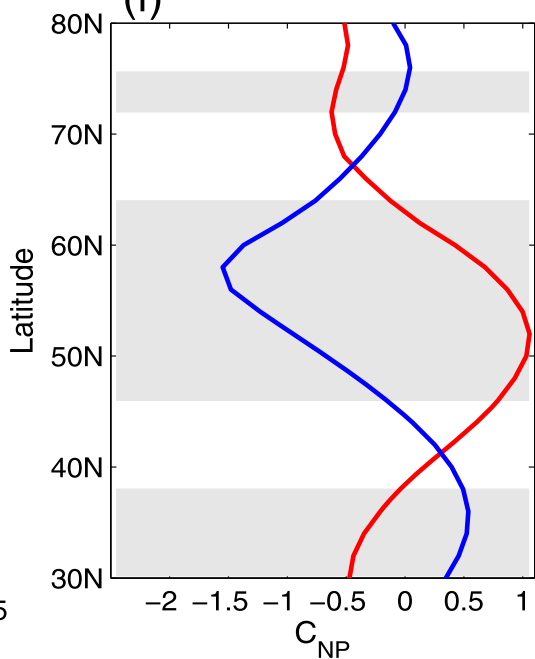

FIG. 11. As in Fig. 8, but for lag -5 to 5 days (mature period).

large-amplitude $\mathrm{NAO}^{-}\left(\mathrm{NAO}^{+}\right)$tends to be strongly nonlinear (linear) process. We further calculate the case of $F=0$ for $R_{d} \rightarrow \infty$. It is found that the above results also hold for such a parameter choice (not shown). The above results also explain why the observed NAO shows strong asymmetry or symmetry breaking between its two phases.

\section{Conclusions and discussion}

In this paper, observational evidence is presented to indicate that there is strong asymmetry of the NAO between its two phases in the amplitude, persistence, and zonal movement. Our study here has revealed that the $\mathrm{NAO}^{-}$has a large amplitude, westward movement, and longer persistence. The opposite feature is seen for the $\mathrm{NAO}^{+}$. By defining and calculating an energy dispersion index, we find that the $\mathrm{NAO}^{+}$shows more intense downstream energy dispersion than the $\mathrm{NAO}^{-}$ especially during the NAO decay phase. It is also seen that blocking days occur more frequently over the North Atlantic (Eurasia) after the $\mathrm{NAO}^{-}\left(\mathrm{NAO}^{+}\right)$peaks, thus suggesting that North Atlantic blocking occurs because of the retrogression of the $\mathrm{NAO}^{-}$, whereas Eurasian blocking occurs because of enhanced downstream energy dispersion of the $\mathrm{NAO}^{+}$.

Motivated by the UNMI model (Luo et al. 2007a,b, 2014, 2015), we have defined dispersion, nonlinear, and zonal movement indices to describe the basic characteristics of the NAO. It is found that the strong asymmetry or symmetry breaking of the NAO is closely related to a large change in the North Atlantic westerly wind between its two phases. In this highly idealized model, because there is $\delta \propto 1 /\left(\beta+F u_{0}\right)(F>0$ and 
(a)

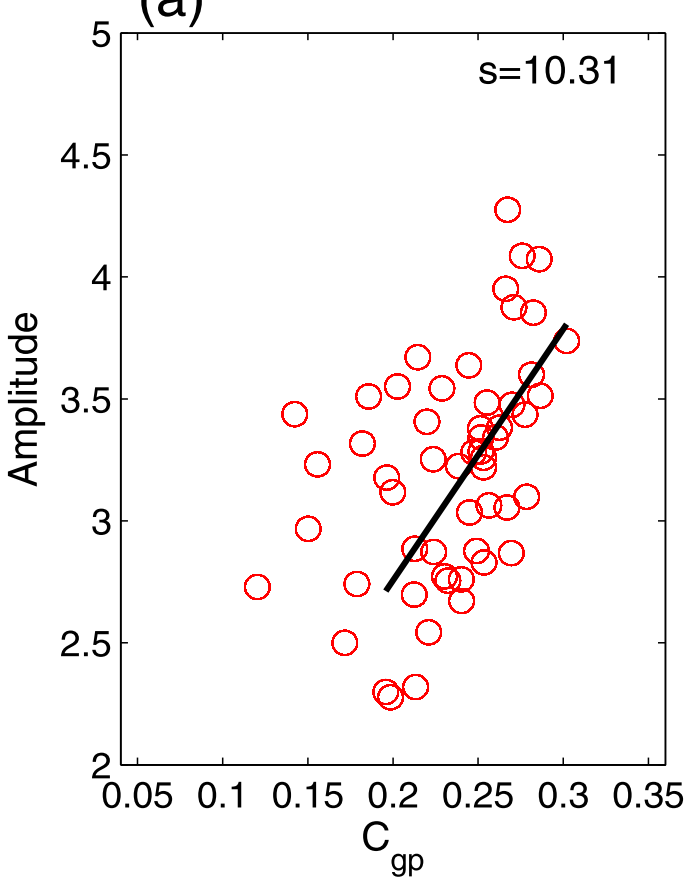

(c)

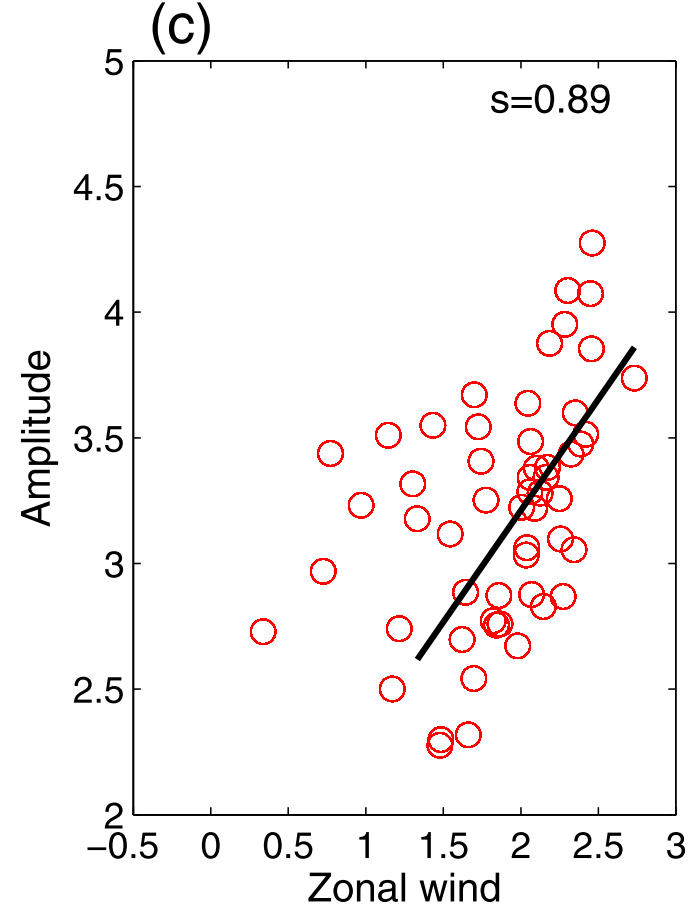

(b)

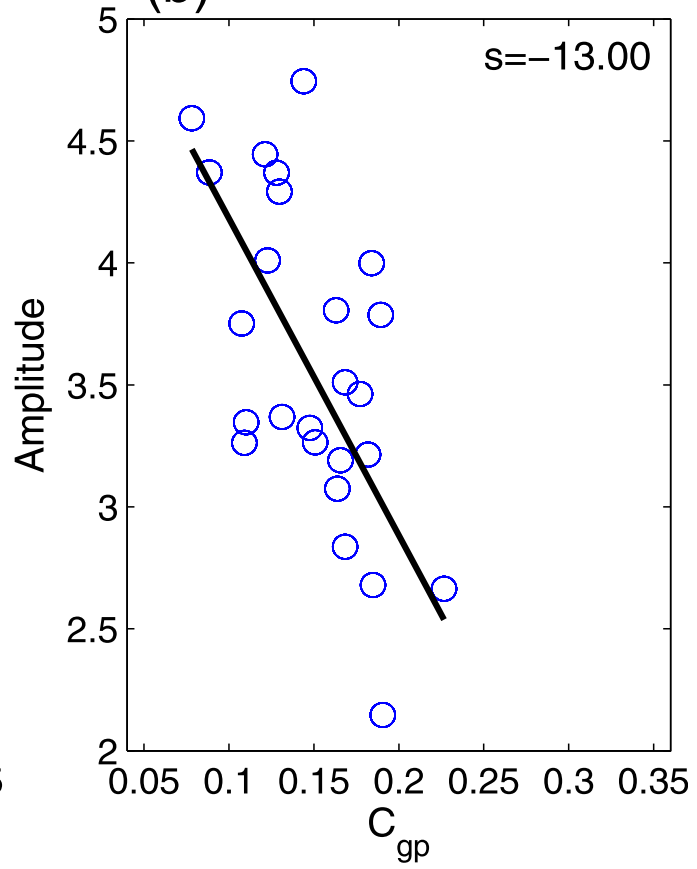

(d)

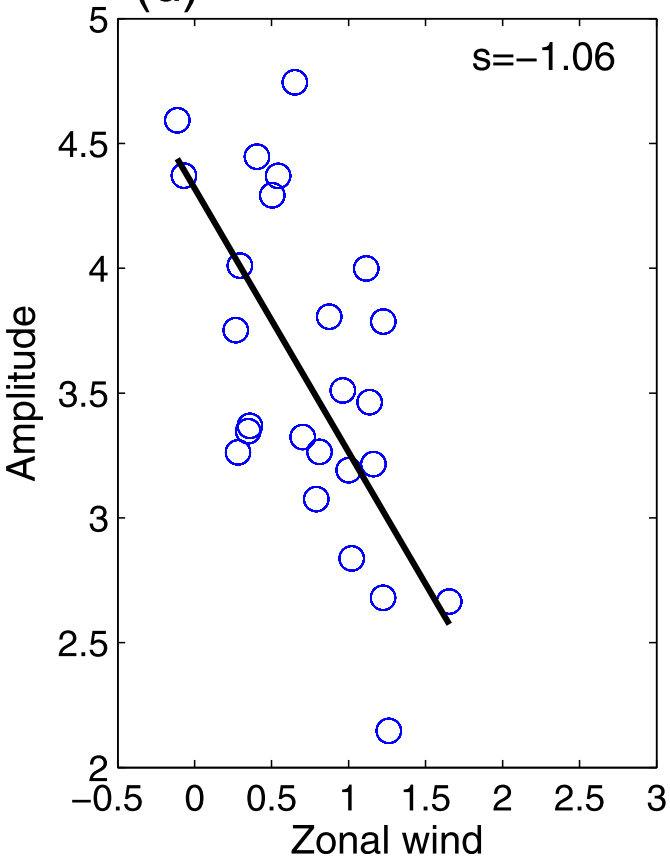

FIG. 12. Scatterplots of the time-mean nondimensional daily NAO amplitude against time-mean, domainaveraged nondimensional (a),(b) $C_{\mathrm{gp}}$ and (c),(d) zonal wind for (a),(c) positive and (b),(d) negative phases during the NAO life cycle. The amplitude of the NAO is defined as the maximum value of the cyclonic (anticyclonic) center for the $\mathrm{NAO}^{+}\left(\mathrm{NAO}^{-}\right)$events. The solid line is the robust fit, and the value of $s$ denotes the slope of the scatterplot, which is statistically significant at the $99 \%$ confidence level for the four cases based on the Student's $t$ test. The area of the domain average used here is over $50^{\circ} \mathrm{W}-0^{\circ}$ and $40^{\circ}-60^{\circ} \mathrm{N}$, whereas the time mean is from lag -5 to 5 days and lag 0 denotes the peak day of the NAO. 
$\left.\beta+F u_{0} \neq 0\right)$ in the nonlinearity coefficient $\delta$ for a uniform basic flow as seen in the expression for $\delta$ in Eq. (3h), the nonlinearity strength of the varying NAO flow should be $\delta \propto 1 /\left(Q_{y}+F u\right)$ if $Q_{y}+F u \neq 0$. On the other hand, because there is $C_{\mathrm{gp}}=C_{g}-C_{p}=2 k^{2}(\beta+$ $\left.F u_{0}\right) /\left(k^{2}+m^{2}+F\right)^{2} \propto \beta+F u_{0}$ for a uniform basic flow, the energy dispersion of the NAO system is positively proportional to $Q_{y}+F u$ for a slowly varying flow.

Under conditions without topography and diabatic heating, the nondimensional barotropic PV is PV = $\beta y+\nabla^{2} \psi-F \psi$. Thus, we obtain the meridional gradient of the $\mathrm{PV}$ as $\mathrm{PV}_{y}=\beta+q_{y}-F u=Q_{y}+F u\left(\mathrm{PV}_{y}=\right.$ $\partial \mathrm{PV} / \partial y)$. The $\mathrm{PV}$ gradient is weak when both $u$ and $Q_{y}$ are small. In this case, we have $C_{\mathrm{gp}} \propto \mathrm{PV}_{y}$ and $\delta \propto 1 / \mathrm{PV}_{y}$. Naturally, the energy dispersion (nonlinearity) of the slowly varying NAO or blocking is proportional to $\mathrm{PV}_{y}$ $\left(1 / \mathrm{PV}_{y}\right.$ for $\left.\mathrm{PV}_{y} \neq 0\right)$. Thus, it is concluded that in the presence of a weak PV gradient, the NAO may be a strongly nonlinear and weakly dispersive system. In contrast, the NAO system can disperse more energy and have a weak nonlinearity when its PV gradient is large. For this case, the NAO system tends to be a linear process. This case is readily seen for the $\mathrm{NAO}^{+}$. For the $\mathrm{NAO}^{-}$or blocking flow, the PV gradient tends to be small or even reversed because of both $Q_{y}$ and $u$ being small. Thus, it is inevitable that the $\mathrm{NAO}^{-}$or blocking flow attains larger amplitude and is long lived because of its weak energy dispersion and strong nonlinearity. This case is also detected under winter Arctic warming conditions because of the small meridional PV gradient associated with the reduced meridional temperature gradient and westerly wind. Further study of this problem will be reported in another paper.

In previous studies (Benedict et al. 2004; Franzke et al. 2004), the $\mathrm{NAO}^{-}$was shown to correspond to cyclonic wave breaking $(\mathrm{CWB})$, and $\mathrm{NAO}^{+}$was shown to correspond to anticyclonic wave breaking (AWB). The CWB (AWB) is seen to have a reduced (enhanced) $\mathrm{PV}_{y}$ (Luo et al. 2008a). As a result, the energy dispersion (nonlinearity) of the $\mathrm{NAO}^{-}$is weakened (intensified) because of its CWB. For this case, the $\mathrm{NAO}^{-}$does not easily disperse its energy when CWB takes place. Instead, its long life can be maintained because of its enhanced nonlinearity and reduced energy dispersion. The opposite case is seen for the $\mathrm{NAO}^{+}$in the presence of AWB. These results suggest that the reduced energy dispersion and intensified nonlinearity of the $\mathrm{NAO}^{-}$are linked to CWB through the reduced PV gradient, whereas the intensified energy dispersion and weakened nonlinearity of the $\mathrm{NAO}^{+}$are linked to AWB through the enhanced PV gradient. Thus, the CWB and AWB are more likely to lead to the asymmetry of the NAO between both phases in persistence and amplitude, rather than the generation origins of the $\mathrm{NAO}^{-}$and $\mathrm{NAO}^{+}$.
The North Atlantic westerly wind is intensified (weakened) and shifted northward (southward) to enhance (reduce) the meridional $\mathrm{PV}$ gradient $\mathrm{PV}_{y}$ as the $\mathrm{NAO}^{+}$ $\left(\mathrm{NAO}^{-}\right)$strengthens. The reduced meridional $\mathrm{PV}$ gradient corresponds to weakened energy dispersion and an intensified nonlinearity of the $\mathrm{NAO}^{-}$that favor largeamplitude and long-lived $\mathrm{NAO}^{-}$events by suppressing its energy dispersion. The westward movement of the $\mathrm{NAO}^{-}$is also seen to be related to the reduced North Atlantic westerly wind. The opposite features are seen for the $\mathrm{NAO}^{+}$. Because the $\mathrm{NAO}^{+}$has much stronger downstream energy dispersion than the $\mathrm{NAO}^{-}$, it tends to be a linear process. Naturally, we conclude that the large difference of the North Atlantic westerly wind strength between the positive and negative NAO phases is likely the main cause of the symmetry breaking of the NAO. It is further found that the nonlinear phase speed $C_{\mathrm{NP}}$ of the $\mathrm{NAO}^{-}\left(\mathrm{NAO}^{+}\right)$is largely negative (positive) in mid- to high latitudes because of the weakened (intensified) North Atlantic westerly wind, thus explaining why the $\mathrm{NAO}^{-}\left(\mathrm{NAO}^{+}\right)$undergoes a marked westward (eastward) movement. In short, the $\mathrm{NAO}^{+}$ $\left(\mathrm{NAO}^{-}\right.$) tends to be a linear (nonlinear) process during its life cycle, and their zonal wind difference leads to a strong asymmetry of the NAO between its two phases.

Moreover, because the change in the North Atlantic westerly wind is closely related to the NAO itself, one can infer that the symmetry breaking of the NAO between its two phases is a natural outcome of the NAO evolution. The above results are based on reanalysis data. However, it must be pointed out that the results obtained here are based on a highly idealized model that excludes the effects of baroclinicity and stratification. Whether these conclusions are held in complex models deserves further investigation even though barotropic processes are dominant for the NAO. This problem will be further examined in the future.

Acknowledgments. The authors acknowledge the support from the National Natural Science Foundation of China (Grants 41430533 and 41790473), National Key Research and Development Program of China (2016YFA0601802), and National Science Foundation Grants AGS-1401220 and OPP-1723832. The authors thank three anonymous reviewers for their helpful suggestions in improving this paper.

\section{APPENDIX}

\section{Dynamical Meaning of the Energy Dispersion Index}

In this appendix, we describe the dynamical basis of the energy dispersion index $D_{I}=\left(H_{A}-H_{D}\right) / H_{A}$ in Fig. 4. This index is defined based on the relative magnitudes of 


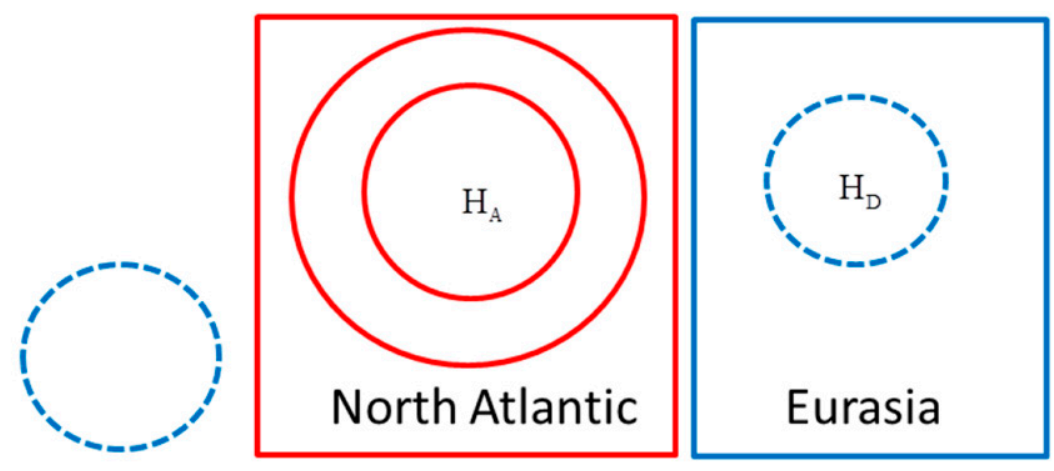

(a)

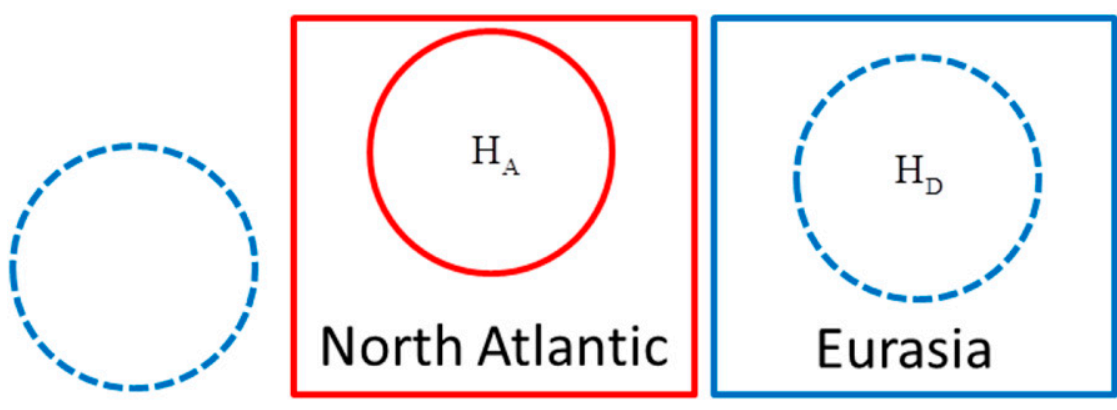

(b)

FIG. A1. Schematic diagram of the construction of the energy dispersion index $D_{I}=$ $\left(H_{A}-H_{D}\right) / H_{A}$ of the $\mathrm{NAO}^{-}$pattern for (a) weak and (b) strong dispersion cases, where $H_{A}$ denotes the absolute value of the anticyclonic anomaly averaged over an area of $4^{\circ}$ latitude $\times 4^{\circ}$ longitude around its maximum center of the northern pole (red circle) of the $\mathrm{NAO}^{-}$in the North Atlantic (red box) and $H_{D}$ denotes the absolute value of the corresponding domain-averaged height anomaly (blue dashed circle) in the downstream side (Eurasia) (blue box) of the North Atlantic.

the anticyclonic height anomaly (the absolute value of $H_{A}$ ) averaged over a domain with $4^{\circ}$ latitude $\times$ $4^{\circ}$ longitude centered on the northern center of the $\mathrm{NAO}^{-}$pattern in the North Atlantic and the same size domain-averaged cyclonic height anomaly (the absolute value $H_{D}$ ) over its downstream side (Eurasia) if the $\mathrm{NAO}^{-}$has a wave train structure as shown in Fig. A1a, similar to the composite result (Fig. 1b).

Below, we will demonstrate that it is reasonable to define $D_{I}$ as an index describing whether the $\mathrm{NAO}^{-}$has strong energy dispersion. For the case without forcing, Eq. (3h) has a solution (Luo 2000):

$$
B=B_{0} \operatorname{sech}\left[\sqrt{\frac{\delta}{2 \lambda}} B_{0}\left(x-C_{g} t\right)\right] \exp \left(i \frac{\delta}{2} B_{0}^{2} t\right),
$$

where $B_{0}$ is the constant amplitude at $(x, t)=(0,0)$

From Eq. (3c), the streamfunction anomaly of the NAO can be obtained as

$$
\begin{aligned}
\psi_{\mathrm{NAO}}= & B_{0} \sqrt{\frac{2}{L_{y}}} \operatorname{sech}\left[\sqrt{\frac{\delta}{2 \lambda}} B_{0}\left(x-C_{g} t\right)\right] \\
& \times \exp \left[i\left(k x-\omega t+\frac{\delta B_{0}^{2} t}{2}\right)\right] \sin (m y)+\mathrm{cc},
\end{aligned}
$$

where $\operatorname{sech}(\Theta)=2 /\left(e^{\Theta}+e^{-\Theta}\right)$ for any variable $\Theta$.

Here, we consider the case of the $\mathrm{NAO}^{-}$. Equation (A2) shows that $\psi_{\mathrm{NAO}}$ tends to decay from $x=0$ as $\delta / \lambda$ is positive. In this case, the $\mathrm{NAO}^{-}$energy is mainly concentrated in a zonal region around $x=0$. Thus, there is $H_{A}>H_{D}$ in Fig. A1a so that $D_{I}$ is positive. Especially, 
when $D_{I}$ is largely positive, we have $H_{A} \gg H_{D}$. For this case, the $\mathrm{NAO}^{-}$has a very large amplitude near $x=$ 0 in the North Atlantic, thus suggesting that the $\mathrm{NAO}^{-}$ is weakly dispersive or even nondispersive. However, when $\delta / \lambda \rightarrow 0$, the streamfunction anomaly of the $\mathrm{NAO}^{-}$has almost the same amplitudes in the North Atlantic and its downstream region (Eurasia) because of $\operatorname{sech}\left[(\delta / 2 \lambda)^{1 / 2} B_{0}\left(x-C_{g} t\right)\right] \rightarrow 1$, thus indicating that the $\mathrm{NAO}^{-}$has strong dispersion. For this case, there is $H_{A} \sim H_{D}$ so that $D_{I}$ is small (Fig. A1b). We also see that $D_{I}$ is negative when $H_{A}<H_{D}$. Thus, the small or negative value of $D_{I}$ implies that the $\mathrm{NAO}^{-}$is strongly dispersive. The value of $D_{I}$ has the same physical meaning for the $\mathrm{NAO}^{+}$as well. In summary, the value of $D_{I}$ can capture the energy dispersion of wave trains related to the NAO.

\section{REFERENCES}

Ambaum, M. H., and B. J. Hoskins, 2002: The NAO tropospherestratosphere connection. J. Climate, 15, 1969-1978, https://doi.org/ 10.1175/1520-0442(2002)015<1969:TNTSC>2.0.CO;2.

Barnes, E. A., and D. L. Hartmann, 2010: Dynamical feedbacks and the persistence of the NAO. J. Atmos. Sci., 67, 851-865, https://doi.org/10.1175/2009JAS3193.1.

Barnston, A. G., and R. E. Livezey, 1987: Classification, seasonality and persistence of low-frequency atmospheric circulation patterns. Mon. Wea. Rev., 115, 1083-1126, https://doi.org/ 10.1175/1520-0493(1987)115<1083:CSAPOL $>2.0 . C O ; 2$.

Baxter, S., and S. Nigam, 2013: The subseasonal teleconnection analysis: PNA development and its relationship to the NAO. J. Climate, 26, 6733-6741, https://doi.org/10.1175/JCLI-D-1200426.1.

Benedict, J., S. Lee, and S. B. Feldstein, 2004: Synoptic view of the North Atlantic Oscillation. J. Atmos. Sci., 61, 121-144, https:// doi.org/10.1175/1520-0469(2004)061<0121:SVOTNA > 2.0.CO;2.

Cassou, C., L. Terray, J. Hurrell, and C. Deser, 2004: North Atlantic winter climate regimes: Spatial asymmetry, stationarity with time, and oceanic forcing. J. Climate, 17, 1055-1068, https://doi.org/ 10.1175/1520-0442(2004)017<1055:NAWCRS > 2.0.CO;2.

Croci-Maspoli, M., C. Schwierz, and H. C. Davies, 2007: Atmospheric blocking: Space-time links to the NAO and PNA. Climate Dyn., 29, 713-725, https://doi.org/10.1007/s00382-007-0259-4.

Dai, G., M. Mu, and Z. Jiang, 2016: Relationships between optimal precursors triggering NAO onset and optimally growing initial errors during NAO prediction. J. Atmos. Sci., 73, 293-317, https://doi.org/10.1175/JAS-D-15-0109.1.

Davini, P., C. Cagnazzo, S. Gualdi, and A. Navarra, 2012: Bidimensional diagnostics, variability, and trends of Northern Hemisphere blocking. J. Climate, 25, 6496-6509, https:// doi.org/10.1175/JCLI-D-12-00032.1.

Dee, D. P., and Coauthors, 2011: The ERA-Interim reanalysis: Configuration and performance of the data assimilation system. Quart. J. Roy. Meteor. Soc., 137, 553-597, https://doi. org/10.1002/qj.828.

Diao, Y., J. Li, and D. Luo, 2006: A new blocking index and its application: Blocking action in the Northern Hemisphere. J. Climate, 19, 4819-4839, https://doi.org/10.1175/JCLI3886.1.

Exner, F. M., 1913: Ubermonatliche Witterungsanomalien auf der nordlichen Erdhalfte im winter, Sitzungsberichte d. Kaiserl. Akad. Wiss., 122, 1165-1241.
Feldstein, S. B., 2003: The dynamics of NAO teleconnection pattern growth and decay. Quart. J. Roy. Meteor. Soc., 129, 901-924, https://doi.org/10.1256/qj.02.76.

Franzke, C., S. Lee, and S. B. Feldstein, 2004: Is the North Atlantic Oscillation a breaking wave? J. Atmos. Sci., 61, 145-160, https:// doi.org/10.1175/1520-0469(2004)061<0145:ITNAOA > 2.0.CO;2.

Haines, K., and J. C. Marshall, 1987: Eddy-forced coherent structures as a prototype of atmospheric blocking. Quart. J. Roy. Meteor. Soc., 113, 681-704, https://doi:10.1002/qj.49711347613.

Hurrell, J. W., 1995: Transient eddy forcing of the rotational flow during northern winter. J. Atmos. Sci., 52, 2286-2301, https:// doi.org/10.1175/1520-0469(1995)052<2286:TEFOTR>2.0.CO;2.

_-, Y. Kushnir, G. Ottersen, and M. Visbeck, Eds., 2003: The North Atlantic Oscillation: Climate Significance and Environmental Impact. Geophys. Monogr., Vol. 134, Amer. Geophys. Union, $279 \mathrm{pp}$.

Jiang, Z. N., M. Mu, and D. H. Luo, 2013: A study of the North Atlantic Oscillation using conditional nonlinear optimal perturbation. J. Atmos. Sci., 70, 855-875, https://doi.org/10.1175/ JAS-D-12-0148.1.

Jin, F. F., L. L. Pan, and M. Watanabe, 2006: Dynamics of synoptic eddy and low-frequency flow interaction. Part I: A linear closure. J. Atmos. Sci., 63, 1677-1694, https://doi.org/10.1175/JAS3715.1.

Jung, T., M. Hilmer, E. Ruprecht, S. Kleppek, S. K. Gulev, and O. Zolina, 2003: Characteristics of the recent eastward shift of interannual NAO variability. J. Climate, 16, 3371-3382, https://doi.org/10.1175/1520-0442(2003)016<3371: COTRES $>2.0 . \mathrm{CO} ; 2$.

Kutzbach, J. E., 1970: Large-scale features of monthly mean Northern Hemisphere anomaly maps of sea-level pressure. Mon. Wea. Rev., 98, 708-716, https://doi.org/10.1175/15200493(1970)098<0708:LSFOMM > 2.3.CO;2.

Lorenz, E. N., 1951: Seasonal and irregular variations of the Northern Hemisphere sea-level pressure profile. J. Meteor., 8, 52-59, https://doi.org/10.1175/1520-0469(1951)008<0052: SAIVOT $>2.0 . \mathrm{CO} ; 2$.

Luo, D., 2000: Planetary-scale baroclinic envelope Rossby solitons in a two-layer model and their interaction with synoptic-scale eddies. Dyn. Atmos. Oceans, 32, 27-74, https://doi.org/ 10.1016/S0377-0265(99)00018-4.

_ 2005: A barotropic envelope Rossby soliton model for blockeddy interaction. Part I: Effect of topography. J. Atmos. Sci., 62, 5-21, https://doi.org/10.1175/1186.1.

-_, and J. Li, 2000: Barotropic interaction between planetary- and synoptic-scale waves during the life cycle of blockings. Adv. Atmos. Sci., 17, 649-670, https://doi.org/10.1007/ s00376-000-0026-5.

_- F. Huang, and Y. Diao, 2001: Interaction between antecedent planetary-scale envelope soliton blocking anticyclone and synoptic-scale eddies: Observations and theory. J. Geophys. Res., 106, 31 795-31 816, https://doi.org/10.1029/2000JD000086.

_ A. R. Lupo, and H. Wan, 2007a: Dynamics of eddy-driven low-frequency dipole modes. Part I: A simple model of North Atlantic Oscillations. J. Atmos. Sci., 64, 3-28, https://doi.org/ 10.1175/JAS3818.1.

— low-frequency dipole modes. Part II: Free mode characteristics of NAO and diagnostic study. J. Atmos. Sci., 64, 29-51, https://doi.org/10.1175/JAS3820.1.

- $\longrightarrow$, and Y. Diao, 2007c: Dynamics of eddy-driven lowfrequency dipole modes. Part III: Meridional displacement of westerly jet anomalies during two phases of NAO. J. Atmos. Sci., 64, 3232-3248, https://doi.org/10.1175/JAS3998.1. 
- — - and $\longrightarrow$, 2008a: Dynamics of eddy-driven lowfrequency dipole modes. Part IV: Planetary and synoptic wave breaking processes during the NAO life cycle. J. Atmos. Sci., 65, 737-765, https://doi.org/10.1175/2007JAS2440.1.

$\longrightarrow, \ldots$, and L. Zhong, 2008b: Dynamical relationship between the phase of North Atlantic Oscillations and meridional excursion of a preexisting jet: An analytical study. J. Atmos. Sci., 65, 1838-1858, https://doi.org/10.1175/2007JAS2560.1.

—, Y. Diao, and S. B. Feldstein, 2011: The variability of the Atlantic storm track activity and North Atlantic Oscillations: A link between intraseasonal and interannual variability. J. Atmos. Sci., 68, 577-601, https://doi.org/10.1175/2010JAS3579.1.

— J. Cha, and S. Feldstein, 2012: Weather regime transitions and the interannual variability of the North Atlantic Oscillation. Part II: Dynamical processes. J. Atmos. Sci., 69, 2347 2363, https://doi.org/10.1175/JAS-D-11-0290.1.

,-- L. Zhong, and A. Dai, 2014: A nonlinear multiscale interaction model for atmospheric blocking: The eddyblocking matching mechanism. Quart. J. Roy. Meteor. Soc., 140, 1785-1808, https://doi.org/10.1002/qj.2337.

_ _ L. Zhong, and C. L. E. Franzke, 2015: Inverse energy cascades in an eddy-induced NAO-type flow: Scale interaction mechanism. J. Atmos. Sci., 72, 3417-3448, https://doi.org/10.1175/JASD-15-0062.1.

Mu, M., and Z. Jiang, 2008: A method to find perturbations that trigger blocking onset: Conditional nonlinear optimal perturbations. J. Atmos. Sci., 65, 3935-3946, https://doi.org/10.1175/ 2008JAS2621.1.

Namias, J., 1950: The index cycle and its role in the general circulation. J. Meteor., 7, 130-139, https://doi.org/10.1175/15200469(1950)007<0130:TICAIR>2.0.CO;2.

O'Reilly, C. H., S. Minobe, A. K. Yoshida, and T. Woollings, 2017: The Gulf Stream influence on wintertime North Atlantic jet variability. Quart. J. Roy. Meteor. Soc., 143, 173-183, https:// doi.org/10.1002/qj.2907.

Palmer, T. N., and J. Räisänen, 2002: Quantifying the risk of extreme seasonal precipitation events in a changing climate. Nature, 415, 512-514, https://doi.org/10.1038/415512a.

Peterson, K. A., J. Lu, and R. J. Greatbatch, 2003: Evidence of nonlinear dynamics in the eastward shift of the NAO. Geophys. Res. Lett., 30, 1030, https://doi.org/10.1029/2002GL015585.

Rivière, G., and I. Orlanski, 2007: Characteristics of the Atlantic storm-track eddy activity and its relation with the North Atlantic Oscillation. J. Atmos. Sci., 64, 241-266, https://doi.org/ 10.1175/JAS3850.1.

Robinson, W. A., 2000: A baroclinic mechanism for the eddy feedback on the zonal index. J. Atmos. Sci., 57, 415-422, https://doi.org/ 10.1175/1520-0469(2000)057<0415:ABMFTE > 2.0.CO;2.

Rodwell, M. J., D. P. Rowell, and C. K. Folland, 1999: Oceanic forcing of the wintertime North Atlantic Oscillation and European climate. Nature, 398, 320-323, https://doi.org/10.1038/18648.

Rogers, J. C., 1997: North Atlantic storm track variability and its association to the North Atlantic Oscillation and climate variability of northern Europe. J. Climate, 10, 1635-1645, https://doi.org/10.1175/1520-0442(1997)010<1635: NASTVA $>2.0 . \mathrm{CO} ; 2$.

Scherrer, S. C., M. Croci-Maspoli, C. Schwierz, and C. Appenzeller, 2006: Two-dimensional indices of atmospheric blocking and their statistical relationship with winter climate patterns in the Euro-Atlantic region. Int. J. Climatol., 26, 233-250, https:// doi.org/10.1002/joc.1250.

Shutts, G. J., 1983: The propagation of eddies in diffluent jetstreams: Eddy vorticity forcing of blocking flow fields. Quart. J. Roy. Meteor. Soc., 109, 737-761, https://doi.org/10.1002/ qj. 49710946204.

Song, J., 2016: Understanding anomalous eddy vorticity forcing in North Atlantic Oscillation events. J. Atmos. Sci., 73, 29853007, https://doi.org/10.1175/JAS-D-15-0253.1.

Strong, C., and R. E. Davis, 2008: Variability in the position and strength of winter jet stream cores related to Northern Hemisphere teleconnections. J. Climate, 21, 584-592, https:// doi.org/10.1175/2007JCLI1723.1.

Tibaldi, S., and F. Molteni, 1990: On the operational predictability of blocking. Tellus, 42A, 343-365, https://doi.org/10.3402/ tellusa.v42i3.11882.

Ulbrich, U., and M. Christoph, 1999: A shift of the NAO and increasing storm track activity over Europe due to anthropogenic greenhouse gas forcing. Climate Dyn., 15, 551-559, https://doi.org/10.1007/s003820050299.

Vallis, G. K., E. P. Gerber, P. J. Kushner, and B. A. Cash, 2004: A mechanism and simple dynamical model of the North Atlantic Oscillation and annular modes. J. Atmos. Sci., 61, 264-280, https:// doi.org/10.1175/1520-0469(2004)061<0264:AMASDM>2.0.CO;2.

Walker, G. T., 1923: Correlation in seasonal variation of weather. VIII, a preliminary study of world weather. Mem. Indian Meteor. Dept., 24, 75-131.

Wang, Y., and G. Magnusdottir, 2012: The shift of the northern node of the NAO and cyclonic Rossby wave breaking. J. Climate, 25, 7973-7982, https://doi.org/10.1175/JCLI-D-11-00596.1.

Watanabe, M., 2004: Asian jet waveguide and a downstream extension of the North Atlantic Oscillation. J. Climate, 17, 46744691, https://doi.org/10.1175/JCLI-3228.1.

Woollings, T., B. Hoskins, M. Blackburn, and P. Berrisford, 2008: A new Rossby wave-breaking interpretation of the North Atlantic Oscillation. J. Atmos. Sci., 65, 609-626, https:// doi.org/10.1175/2007JAS2347.1.

_- A. Hannachi, and B. Hoskins, 2010: Variability of the North Atlantic eddy-driven jet stream. Quart. J. Roy. Meteor. Soc., 136, 856-868, https://doi.org/10.1002/qj.625.

C. Czuchnicki, and C. Franzke, 2014: Twentieth century North Atlantic jet variability. Quart. J. Roy. Meteor. Soc., 140, 783-791, https://doi.org/10.1002/qj.2197.

Yiou, P., and M. Nogaj, 2004: Extreme climatic events and weather regimes over the North Atlantic: When and where? Geophys. Res. Lett., 31, L07202, https://doi.org/10.1029/ 2003 GL019119. 